\title{
Ulam stability results to a class of nonlinear implicit boundary value problems of impulsive fractional differential equations
}

\section{A. Ali ${ }^{1 *}$, K. Shah ${ }^{1}$ and D. Baleanu ${ }^{2}$}

\section{"Correspondence:}

arshad.swatpk@gmail.com

'Department of Mathematics,

University of Malakand, $\operatorname{Dir}(\mathrm{L})$,

Khyber Pakhtunkhwa, Pakistan

Full list of author information is

available at the end of the article

\section{Springer}

\begin{abstract}
In this paper, we derive some sufficient conditions which ensure the existence and uniqueness of a solution for a class of nonlinear three point boundary value problems of fractional order implicit differential equations (FOIDEs) with some boundary and impulsive conditions. Also we investigate various types of Hyers-Ulam stability (HUS) for our concerned problem. Using classical fixed point theory and nonlinear functional analysis, we obtain the required conditions. In the last section we give an example to show the applicability of our obtained results.
\end{abstract}

Keywords: Caputo derivative; Boundary conditions; Hyers-Ulam stability

\section{Introduction}

Differential equations of fractional order have been attracted the attention of researchers in the last few decades. It is due to the fact that fractional order derivatives provide power tools for the description of memory and hereditary characteristics of different processes and materials in various fields of science and engineering, (see [1-4]).

The impulsive phenomenon, which is a sudden and discontinuous change, is naturally observed in many physical systems. We model and describe such type of evolutionary processes via differential equations with some impulsive conditions. Significant and enormous number of applications of impulsive differential equations can be traced in mechanics, engineering, medicine, ecology, etc.; see for instance [5-7]. In the literature, the integer-order impulsive differential equations corresponding to initial and boundary conditions have been investigated extensively; see [8-12] and the references cited therein. There are many evolutionary processes related to pharmacotherapy, hemodynamics equilibrium of a person, introduction of bloodstream in the body and problems related to economical and national income, which cannot be accurately described by classical implicit impulsive differential equations. In such a situation the fractional order implicit impulsive differential equations are proved as powerful tools. The existence theory of the aforesaid problems have been extensively addressed in many articles; see $[13,14]$ and the references therein.

On the other side stability analysis, which is so much important from a numerical and optimization point of view, has been attracted the attention of researchers. So far various concepts of stability analysis, including Laypunov stability [15, 16], Mittag-Leffler stability

(c) The Author(s) 2019. This article is distributed under the terms of the Creative Commons Attribution 4.0 International License (http://creativecommons.org/licenses/by/4.0/), which permits unrestricted use, distribution, and reproduction in any medium, provided you give appropriate credit to the original author(s) and the source, provide a link to the Creative Commons license, and indicate if changes were made. 
[17], exponential stability [18] and Hyers-Ulam stability, have been introduced. Among all these concepts, Hyers-Ulam type stability analysis has been considered a relatively easy and simple way of studying the stability of solutions to fractional order implicit differential equations (FOIDEs). Ulam and Hyers introduced this concept of stability analysis in the mid of 19th century for functional problems; see [19, 20]. Many mathematicians generalized this concept in different directions; see [21-25]. For recent contribution on this area we refer to the work in $[26,27]$.

In this paper we study existence and uniqueness of solution as well as stability analysis to the following problem:

$$
\begin{cases}{ }_{0}^{C} \mathrm{D}_{t_{i}}^{\rho} z(t)=\mathrm{F}(t, z(t), & \left.{ }_{0}^{C} \mathrm{D}_{t_{i}}^{\rho} z(t)\right), t \in \mathrm{J}, t \neq t_{i}, i=1,2, \ldots, m, \\ \left.\Delta z(t)\right|_{t=t_{i}}=\mathrm{I}_{i}\left(z\left(t_{i}\right)\right), & \left.\Delta z^{\prime}(t)\right|_{t=t_{i}}=\tilde{\mathrm{I}}_{i}\left(z\left(t_{i}\right)\right), \quad i=1,2, \ldots, m, \\ \left.z(t)\right|_{t=0}=-\left.z^{\prime}(t)\right|_{t=0}, & \left.z(t)\right|_{t=1}=-z^{\prime}(t)_{t=\varrho}, \\ \varrho \in(0,1), \varrho \neq t_{i}, i=1,2, \ldots, m, & \end{cases}
$$

where the notation ${ }_{0}^{C} \mathrm{D}_{t_{i}}^{\rho}$ stands for Caputo fractional derivative of order $\rho \in(1,2], \mathrm{J}=[0,1]$, and $\mathrm{F}: \mathrm{J} \times \mathrm{R}^{2} \rightarrow \mathrm{R}$ is a continuous function. Further, the nonlinear functions $\mathrm{I}_{i}, \tilde{\mathrm{I}}_{i}: \mathrm{R} \rightarrow$ $\mathrm{R}$, are also continuous for $i=1,2, \ldots, m$ and $\left.\Delta z(t)\right|_{t=t_{i}}=z\left(t_{i}^{+}\right)-z\left(t_{i}^{-}\right),\left.\Delta z^{\prime}(t)\right|_{t=t_{i}}=z^{\prime}\left(t_{i}^{+}\right)-$ $z^{\prime}\left(t_{i}^{-}\right)$, where $z\left(t_{i}^{+}\right)$and $z\left(t_{i}^{-}\right)$represent the right and left-hand limit of the function $z(t)$, respectively, at $t=t_{i}$. Also, $0=t_{0}<t_{1}<t_{2}<\cdots<t_{m}<t_{m+1}=1, m \in \mathbb{Z}^{+}$, where $\mathbb{Z}^{+}$is the set of positive integers.

\section{Background materials and some auxiliary results}

We recall some well-known results, definitions and theorems needed in this study. Splitting the interval $J$ into sub intervals $\left[0, t_{1}\right],\left(t_{1}, t_{2}\right],\left(t_{2}, t_{3}\right], \ldots,\left(t_{p-1}, t_{p}\right],\left(t_{m}, 1\right]$, and denote these sub intervals by $\mathrm{J}_{0}, \mathrm{~J}_{1}, \mathrm{~J}_{2}, \ldots, \mathrm{J}_{m-1}, \mathrm{~J}_{m}$, respectively. Let $\mathrm{J}=\mathrm{J} \backslash\left\{t_{1}, t_{2}, t_{3}, \ldots, t_{m}\right\}$. We define the space $\mathrm{E}=P C(\mathrm{~J}, \mathrm{R})=\left\{z: \mathrm{J} \rightarrow \mathrm{R}: z \in C\left(\mathrm{~J}_{i}, \mathrm{R}\right)\right.$, and $z\left(t_{i}^{+}\right), z\left(t_{i}^{-}\right)$exist, for $\left.i=1,2, \ldots, m\right\}$. Obviously $\left(\mathrm{E},\|z\|_{\mathrm{E}}\right)$ is a Banach space with the norm given by $\|z\|_{\mathrm{E}}=\max \{|z(t)|: t \in \mathrm{J}\}$.

Definition 1 ([2]) The Caputo fractional derivative of a function $z:(0, \infty) \rightarrow \mathrm{R}$, is defined as

$$
{ }_{0}^{C} \mathrm{D}_{t}^{\rho} z(t)=\int_{0}^{t} \frac{(t-\xi)^{k-\rho-1}}{\Gamma(k-\rho)} z^{(k)}(\xi) d \xi,
$$

where $k=[\rho]+1$ and $[\rho]$ represents the integer part of the real number $\rho$.

Definition 2 ([3]) The fractional order $(0<\rho<\infty)$ integral of a function $z \in L^{1}\left([0, T], \mathrm{R}^{+}\right)$ is defined as

$$
{ }_{0} I_{t}^{\rho} z(t)=\int_{0}^{t} \frac{(t-\xi)^{\rho-1}}{\Gamma(\rho)} z(\xi) d \xi
$$

such that the right side is point-wise defined on $\mathrm{R}^{+}$.

Lemma 1 ([28]) For $\rho>0$, the given result holds

$$
{ }_{0} I_{t}^{\rho}\left[{ }_{0}^{C} \mathrm{D}_{t}^{\rho} z(t)\right]=z(t)-\sum_{i=0}^{k-1} \frac{z^{(i)}(0)}{i !} t^{i}, \quad \text { where } k=[\rho]+1 .
$$


Lemma 2 ([28]) For $\rho>0$, the differential equation ${ }_{0}^{C} \mathrm{D}_{t}^{\rho} z(t)=h(t)$, has the following solution:

$$
z(t)={ }_{0} I_{t}^{\rho} h(t)+\sum_{i=0}^{k-1} \frac{z^{(i)}(0)}{i !} t^{i}
$$

where $k=[\rho]+1$.

Theorem 1 ([2]) Let $\rho>0$, then

$$
{ }_{0} I_{t}^{\rho}\left[{ }_{0}^{C} \mathrm{D}_{t}^{\rho} z(t)\right]=z(t)+e_{0}+e_{1} t+e_{2} t^{2}+e_{3} t^{3}+\cdots+e_{k-1} t^{k-1},
$$

where $e_{i} \in \mathrm{R}, i=0,1,2,3, \ldots, k-1, k=[\rho]+1$.

We give the following three sets of inequalities.

If $z \in \mathrm{E}$, then, for some constants $\varphi>0, \epsilon>0$ with a nondecreasing function $\theta: \mathrm{J} \rightarrow \mathrm{R}$, the results given below hold for $i=1,2, \ldots, m$ :

$$
\begin{aligned}
& \left\{\begin{array}{l}
\left|{ }_{0}^{C} \mathrm{D}_{t_{i}}^{\rho} z(t)-\mathrm{F}\left(t, z(t),{ }_{0}^{C} \mathrm{D}_{t_{i}}^{\rho} z(t)\right)\right| \leq \epsilon, \quad t \in \mathrm{J}_{i}, \\
|\Delta z(t)|_{t=t_{i}}-\mathrm{I}_{i}\left(z\left(t_{i}\right)\right) \mid \leq \epsilon, \\
\left|\Delta z^{\prime}(t)\right|_{t=t_{i}}-\tilde{\mathrm{I}}_{i}\left(z\left(t_{i}\right)\right) \mid \leq \epsilon,
\end{array}\right. \\
& \left\{\begin{array}{l}
{ }_{0}^{C} \mathrm{D}_{t_{i}}^{\rho} z(t)-\mathrm{F}\left(t, z(t),{ }_{0}^{C} \mathrm{D}_{t_{i}}^{\rho} z(t)\right) \mid \leq \theta(t), \quad t \in \mathrm{J}_{i}, \\
|\Delta z(t)|_{t=t_{i}}-\mathrm{I}_{i}\left(z\left(t_{i}\right)\right) \mid \leq \varphi, \\
\left.\left|\Delta z^{\prime}(t)\right|\right|_{t=t_{i}}-\tilde{\mathrm{I}}_{i}\left(z\left(t_{i}\right)\right) \mid \leq \varphi,
\end{array}\right. \\
& \left\{\begin{array}{l}
{ }_{0}^{C} \mathrm{D}_{t_{i}}^{\rho} z(t)-\mathrm{F}\left(t, z(t),{ }_{0}^{C} \mathrm{D}_{t_{i}}^{\rho} z(t)\right) \mid \leq \epsilon \theta(t), \quad t \in \mathrm{J}_{i}, \\
|\Delta z(t)|_{t=t_{i}}-\mathrm{I}_{i}\left(z\left(t_{i}\right)\right) \mid \leq \epsilon \varphi, \\
\left|\Delta z^{\prime}(t)\right|_{t=t_{i}}-\tilde{\mathrm{I}}_{i}\left(z\left(t_{i}\right)\right) \mid \leq \epsilon \varphi .
\end{array}\right.
\end{aligned}
$$

Definition 3 ([25]) The problem (1) is known to be UH stable if for $\epsilon>0$ there exists a constant $\mathbf{C}_{m, \rho}>0$ such that, for every solution $\bar{z} \in \mathrm{E}$ of the inequality (2), one has a unique solution $z \in \mathrm{E}$ to problem (1) satisfying

$$
|\bar{z}(t)-z(t)| \leq \mathbf{C}_{m, \rho} \epsilon, \quad t \in \mathrm{J} .
$$

Definition 4 ([25]) The problem (1) is known to be GHU stable if for every solution $\bar{z} \in \mathrm{E}$ of the inequality (3) and $\epsilon>0$, with a constant $\psi_{m, \rho} \in C\left(\mathrm{R}^{+}, \mathrm{R}^{+}\right)$, there is unique solution $z \in \mathrm{E}$ of problem (1) satisfying

$$
|\bar{z}(t)-z(t)| \leq \psi_{m, \rho}(\epsilon), \quad t \in \mathrm{J} .
$$

Definition 5 ([25]) The problem (1) is known to be HUR stable corresponding to $(\theta, \varphi)$ if for every $\epsilon>0$ there exists a real number $\mathbf{C}_{m, \rho, \theta}>0$, such that, for any solution $\bar{z} \in \mathrm{E}$ of the inequality (4), one has a unique solution $z \in \mathrm{E}$ of problem (1) satisfying

$$
|\bar{z}(t)-z(t)| \leq \mathbf{C}_{m, \rho, \theta} \epsilon(\theta(t)+\varphi), \quad t \in \mathrm{J} .
$$


Definition 6 ([25]) The problem (1) is known to be GHUR stable with respect to $(\theta, \varphi)$, if there exists a constant $\mathbf{C}_{m, \rho, \theta}>0$, such that, for each solution $\bar{z} \in \mathrm{E}$ of the inequality (3), one has a solution $z \in \mathrm{E}$ of problem (1) satisfying

$$
|\bar{z}(t)-z(t)| \leq \mathbf{C}_{\mathrm{F}, m, \rho, \theta}(\theta(t)+\varphi), \quad t \in \mathrm{J} .
$$

Remark 1 The function $z \in \mathrm{E}$ is called a solution for the inequality (2) if one has a function $\phi \in \mathrm{E}$ together with a sequence $\phi_{i}, i=1,2, \ldots, m$, depending on $z$ such that

(i) $|\phi(t)| \leq \epsilon,\left|\phi_{i}\right| \leq \epsilon, t \in \mathrm{J}_{i}, i=1,2, \ldots, m$;

(ii) ${ }_{0}^{C} \mathrm{D}_{t_{i}}^{\rho} z(t)=\mathrm{F}\left(t, z(t),{ }_{0}^{C} \mathrm{D}_{t_{i}}^{\rho} z(t)\right)+\phi(t), t \in \mathrm{J}_{i}, i=1,2, \ldots, m$;

(iii) $\left.\Delta z(t)\right|_{t=t_{i}}=\mathrm{I}_{i}\left(z\left(t_{i}\right)\right)+\phi_{i}, t \in \mathrm{J}_{i}, i=1,2, \ldots, m$;

(iv) $\left.\Delta z^{\prime}(t)\right|_{t=t_{i}}=\tilde{\mathrm{I}}_{i}\left(z\left(t_{i}\right)\right)+\phi_{i}, t \in \mathrm{J}_{i}, i=1,2, \ldots, m$.

Remark 2 A function $z \in \mathrm{E}$ is a solution of the inequality (4) if one has a function $\phi \in \mathrm{E}$ and a sequence $\phi_{i}, i=1,2, \ldots, m$ depending on $z$ with:

(i) $|\phi(t)| \leq \epsilon \theta(t),\left|\phi_{i}\right| \leq \epsilon \varphi, t \in \mathrm{J}_{i}, i=1,2, \ldots, m$;

(ii) ${ }_{0}^{C} \mathrm{D}_{t_{i}}^{\rho} z(t)=\mathrm{F}\left(t, z(t),{ }_{0}^{C} \mathrm{D}_{t_{i}}^{\rho} z(t)\right)+\phi(t), t \in \mathrm{J}_{i}, i=1,2, \ldots, m$;

(iii) $\left.\Delta z(t)\right|_{t=t_{i}}=\mathrm{I}_{i}\left(z\left(t_{i}\right)\right)+\phi_{i}, t \in \mathrm{J}_{i}, i=1,2, \ldots, m$;

(iv) $\left.\Delta z^{\prime}(t)\right|_{t=t_{i}}=\tilde{\mathrm{I}}_{i}\left(z\left(t_{i}\right)\right)+\phi_{i}, t \in \mathrm{J}_{i}, i=1,2, \ldots, m$.

Similarly one can state such a remark for the inequality (3).

Theorem 2 (Schaefer's fixed point theorem [29]) Let $\mathrm{E}$ be a Banach space and $\mathscr{T}: \mathrm{E} \rightarrow \mathrm{E}$ is completely continuous operator and the set $\mathrm{W}=\{z \in \mathrm{E}: z=\eta \mathscr{T} z, 0<\eta<1\}$ is bounded.

Then $\mathscr{T}$ has a fixed point in $\mathrm{E}$.

Lemma 3 Let $\rho \in(1,2], \sigma: \mathrm{J} \rightarrow \mathrm{R}$ be a continuous function, then the function $z \in \mathrm{E}$ is the solution to the following problem:

$$
\left\{\begin{array}{l}
{ }_{0}^{C} \mathrm{D}_{t_{i}}^{\rho} z(t)=\sigma(t), \quad 0<t<1, t \neq t_{i}, i=1,2, \ldots, m, \\
\left.\Delta z(t)\right|_{t=t_{i}}=\mathrm{I}_{i}\left(z\left(t_{i}\right)\right),\left.\quad \Delta z^{\prime}(t)\right|_{t=t_{i}}=\tilde{\mathrm{I}}_{i}\left(z\left(t_{i}\right)\right), \quad i=1,2, \ldots, m, \\
\left.z(t)\right|_{t=0}=-\left.z^{\prime}(t)\right|_{t=0},\left.\quad z(t)\right|_{t=1}=-z^{\prime}(\varrho), \\
\varrho \in(0,1), \varrho \neq t_{i} \text { for } i=, 1,2, \ldots, m,
\end{array}\right.
$$

if and only if $z$ satisfies the following integral equation:

$$
z(t)=\left\{\begin{array}{l}
\frac{1}{\Gamma(\rho)} \int_{0}^{t}(t-\xi)^{\rho-1} \sigma(\xi) d \xi+\mathcal{A}(1-t), \quad t \in \mathrm{J}_{0} \\
\frac{1}{\Gamma(\rho)} \int_{t_{k}}^{t}(t-\xi)^{\rho-1} \sigma(\xi) d \xi+\frac{1}{\Gamma(\rho)} \sum_{i=1}^{k} \int_{t_{i-1}}^{t_{i}}\left(t_{i}-\xi\right)^{\rho-1} \sigma(\xi) d \xi \\
\quad+\frac{1}{\Gamma(\rho-1)} \sum_{i=1}^{k}\left(t-t_{i}\right) \int_{t_{i-1}}^{t_{i}}\left(t_{i}-\xi\right)^{\rho-2} \sigma(\xi) d \xi+\sum_{i=1}^{k}\left(t-t_{i}\right) \tilde{\mathrm{I}}_{i}\left(z\left(t_{i}\right)\right) \\
\quad+\sum_{i=1}^{k} \mathrm{I}_{i}\left(z\left(t_{i}\right)\right)+\mathcal{A}(1-t), \quad t \in \mathrm{J}_{k}, k=1,2,3, \ldots, m,
\end{array}\right.
$$


where

$$
\begin{aligned}
\mathcal{A}= & \left.\left.\frac{1}{\Gamma(\rho)} \sum_{i=1}^{m+1} \int_{t_{i-1}}^{t_{i}}\left(t_{i}-\xi\right)^{\rho-1} \sigma(\xi)\right) d \xi+\frac{1}{\Gamma(\rho-1)} \sum_{i=1}^{m}\left(1-t_{i}\right) \int_{t_{i-1}}^{t_{i}}\left(t_{i}-\xi\right)^{\rho-2} \sigma(\xi)\right) d \xi \\
& +\frac{1}{\Gamma(\rho-1)} \int_{t_{i}}^{\varrho}(\varrho-\xi)^{\rho-2} \sigma(\xi) d \xi+\frac{1}{\Gamma(\rho-1)} \sum_{i=1}^{n} \int_{t_{i-1}}^{t_{i}}\left(t_{i}-\xi\right)^{\rho-2} \sigma(\xi) d \xi \\
& +\sum_{i=1}^{m}\left(1-t_{i}\right) \tilde{\mathrm{I}}_{i}\left(z\left(t_{i}\right)\right)+\sum_{i=1}^{n} \tilde{\mathrm{I}}_{i}\left(z\left(t_{i}\right)\right)+\sum_{i=1}^{m} \mathrm{I}_{i}\left(z\left(t_{i}\right)\right) .
\end{aligned}
$$

Proof Assume that, for $t \in \mathrm{J}_{0}, z$ is a solution of (5). Then, by Lemma 1, there exist $a_{1}, a_{2} \in \mathrm{R}$ such that

$$
z(t)={ }_{0} I_{t}^{\rho} \sigma(t)-a_{1}-a_{2} t=\frac{1}{\Gamma(\rho)} \int_{0}^{t}(t-\xi)^{\rho-1} \sigma(\xi) d \xi-a_{1}-a_{2} t
$$

which also yields

$$
z^{\prime}(t)=\frac{1}{\Gamma(\rho-1)} \int_{0}^{t}(t-\xi)^{\rho-2} \sigma(\xi) d \xi-a_{2} .
$$

Let for $t \in \mathrm{J}_{1}$, we have $d_{1}, d_{2} \in \mathrm{R}$, with

$$
\begin{aligned}
& z(t)=\frac{1}{\Gamma(\rho)} \int_{t_{1}}^{t}(t-\xi)^{\rho-1} \sigma(\xi) d \xi-d_{1}-d_{2}\left(t-t_{1}\right), \\
& z^{\prime}(t)=\frac{1}{\Gamma(\rho-1)} \int_{t_{1}}^{t}(t-\xi)^{\rho-2} \sigma(\xi) d \xi-d_{2}
\end{aligned}
$$

This leads to

$$
\begin{aligned}
& z\left(t_{1}^{-}\right)=\frac{1}{\Gamma(\rho)} \int_{t_{0}}^{t_{1}}\left(t_{1}-\xi\right)^{\rho-1} \sigma(\xi) d \xi-a_{1}-a_{2} t_{1}, \quad z\left(t_{1}^{+}\right)=-d_{1}, \\
& z^{\prime}\left(t_{1}^{-}\right)=\frac{1}{\Gamma(\rho-1)} \int_{0}^{t_{1}}\left(t_{1}-\xi\right)^{\rho-2} \sigma(\xi) d \xi-a_{2}, \quad z^{\prime}\left(t_{1}^{+}\right)=-d_{2} .
\end{aligned}
$$

Due to impulsive conditions, we have

$$
\Delta z\left(t_{1}\right)=z\left(t_{1}^{+}\right)-z\left(t_{1}^{-}\right)=\mathrm{I}_{1}\left(z\left(t_{1}\right)\right) \quad \text { and } \quad \Delta z^{\prime}\left(t_{1}\right)=z^{\prime}\left(t_{1}^{+}\right)-z^{\prime}\left(t_{1}^{-}\right)=\tilde{\mathrm{I}}_{1}\left(z\left(t_{1}\right)\right) \text {, }
$$

we have

$$
\begin{aligned}
& -d_{1}=\frac{1}{\Gamma(\rho)} \int_{t_{0}}^{t_{1}}\left(t_{1}-\xi\right)^{\rho-1} \sigma(\xi) d \xi-a_{1}-a_{2} t_{1}+\mathrm{I}_{1}\left(z\left(t_{1}\right)\right), \\
& -d_{2}=\frac{1}{\Gamma(\rho-1)} \int_{0}^{t_{1}}\left(t_{1}-\xi\right)^{\rho-2} \sigma(\xi) d \xi-a_{2}+\tilde{\mathrm{I}}_{1}\left(z\left(t_{1}\right)\right) .
\end{aligned}
$$


Thus (9) implies

$$
\begin{aligned}
z(t)= & \frac{1}{\Gamma(\rho)} \int_{t_{1}}^{t}(t-\xi)^{\rho-1} \sigma(\xi) d \xi+\frac{1}{\Gamma(\rho)} \int_{0}^{t_{1}}\left(t_{1}-\xi\right)^{\rho-1} \sigma(\xi) d \xi \\
& +\frac{t-t_{1}}{\Gamma(\rho-1)} \int_{0}^{t_{1}}\left(t_{1}-\xi\right)^{\rho-2} \sigma(\xi) d \xi+\mathrm{I}_{i}\left(z\left(t_{1}\right)\right)+\left(t-t_{1}\right) \tilde{\mathrm{I}}_{1}\left(z\left(t_{1}\right)\right) \\
& -a_{1}-a_{2} t, \quad t \in \mathrm{J}_{1} .
\end{aligned}
$$

Similarly for $t \in \mathrm{J}_{k}$, one has

$$
\begin{aligned}
z(t)= & \frac{1}{\Gamma(\rho)} \int_{t_{k}}^{t}(t-\xi)^{\rho-1} \sigma(\xi) d \xi+\frac{1}{\Gamma(\rho)} \sum_{i=1}^{k} \int_{t_{i-1}}^{t_{i}}\left(t_{i}-\xi\right)^{\rho-1} \sigma(\xi) d \xi \\
& +\frac{1}{\Gamma(\rho-1)} \sum_{i=1}^{k}\left(t-t_{i}\right) \int_{t_{i-1}}^{t_{i}}\left(t_{i}-\xi\right)^{\rho-2} \sigma(\xi) d \xi+\sum_{i=1}^{k}\left(t-t_{i}\right) \tilde{\mathrm{I}}_{i}\left(z\left(t_{i}\right)\right) \\
& +\sum_{i=1}^{k} \mathrm{I}_{i}\left(z\left(t_{i}\right)\right)-a_{1}-a_{2} t, \quad t \in \mathrm{J}_{k}, k=1,2, \ldots, m .
\end{aligned}
$$

Using the given boundary conditions in (7), (8) and (10), we obtain $a_{1}+a_{2}=0$ and

$$
\begin{aligned}
z(1)= & \frac{1}{\Gamma(\rho)} \int_{t_{m}}^{1}(1-\xi)^{\rho-1} \sigma(\xi) d \xi+\frac{1}{\Gamma(\rho)} \sum_{i=1}^{m} \int_{t_{i-1}}^{t_{i}}\left(t_{i}-\xi\right)^{\rho-1} \sigma(\xi) d \xi \\
& +\frac{1}{\Gamma(\rho-1)} \sum_{i=1}^{m}\left(1-t_{i}\right) \int_{t_{i-1}}^{t_{i}}\left(t_{i}-\xi\right)^{\rho-2} \sigma(\xi) d \xi+\sum_{i=1}^{m}\left(1-t_{i}\right) \tilde{\mathrm{I}}_{i}\left(z\left(t_{i}\right)\right) \\
& +\sum_{i=1}^{m} \mathrm{I}_{i}\left(z\left(t_{i}\right)\right)-a_{1}-a_{2}, \\
z^{\prime}(\varrho)= & \frac{1}{\Gamma(\rho-1)} \int_{t_{n}}^{\varrho}(\varrho-\xi)^{\rho-2} \sigma(\xi) d \xi+\frac{1}{\Gamma(\rho-1)} \sum_{i=1}^{n} \int_{t_{i-1}}^{t_{i}}\left(t_{i}-\xi\right)^{\rho-2} \sigma(\xi) d \xi \\
& +\sum_{i=1}^{n} \tilde{\mathrm{I}}_{i}\left(z\left(t_{i}\right)\right)-a_{2} .
\end{aligned}
$$

Therefore, in view of $z(1)=-z^{\prime}(\varrho)$ and $a_{1}+a_{2}=0$, we get

$$
\begin{aligned}
a_{1}= & -\frac{1}{\Gamma(\rho)} \sum_{i=1}^{m+1} \int_{t_{i-1}}^{t_{i}}\left(t_{i}-\xi\right)^{\rho-1} \sigma(\xi) d \xi-\frac{1}{\Gamma(\rho-1)} \sum_{i=1}^{m}\left(1-t_{i}\right) \int_{t_{i-1}}^{t_{i}}\left(t_{i}-\xi\right)^{\rho-2} \sigma(\xi) d \xi \\
& -\frac{1}{\Gamma(\rho-1)} \int_{t_{i}}^{\varrho}(\varrho-\xi)^{\rho-2} \sigma(\xi) d \xi-\frac{1}{\Gamma(\rho-1)} \sum_{i=1}^{n} \int_{t_{i-1}}^{t_{i}}\left(t_{i}-\xi\right)^{\rho-2} \sigma(\xi) d \xi \\
& -\sum_{i=1}^{m}\left(1-t_{i}\right) \tilde{\mathrm{I}}_{i}\left(z\left(t_{i}\right)\right)-\sum_{i=1}^{n} \tilde{\mathrm{I}}_{i}\left(z\left(t_{i}\right)\right)-\sum_{i=1}^{m} \mathrm{I}_{i}\left(z\left(t_{i}\right)\right)
\end{aligned}
$$




$$
\begin{aligned}
a_{2}= & \frac{1}{\Gamma(\rho)} \sum_{i=1}^{m+1} \int_{t_{i-1}}^{t_{i}}\left(t_{i}-\xi\right)^{\rho-1} \sigma(\xi) d \xi+\frac{1}{\Gamma(\rho-1)} \sum_{i=1}^{m}\left(1-t_{i}\right) \int_{t_{i-1}}^{t_{i}}\left(t_{i}-\xi\right)^{\rho-2} \sigma(\xi) d \xi \\
& +\frac{1}{\Gamma(\rho-1)} \int_{t_{i}}^{\varrho}(\varrho-\xi)^{\rho-2} \sigma(\xi) d \xi+\frac{1}{\Gamma(\rho-1)} \sum_{i=1}^{m} \int_{t_{i-1}}^{t_{i}}\left(t_{i}-\xi\right)^{\rho-2} \sigma(\xi) d \xi \\
& +\sum_{i=1}^{m}\left(1-t_{i}\right) \tilde{\mathrm{I}}_{i}\left(z\left(t_{i}\right)\right)+\sum_{i=1}^{n} \tilde{\mathrm{I}}_{i}\left(z\left(t_{i}\right)\right)+\sum_{i=1}^{m} \mathrm{I}_{i}\left(z\left(t_{i}\right)\right) .
\end{aligned}
$$

Inserting these values of $a_{1}$ and $a_{2}$ in (7) and (10), respectively, with $\mathcal{A}=a_{2}$, we get (6). Conversely if (6) has a solution $z$, then it is obvious that the solution $z(t)$ satisfies problem (5) under the given conditions.

Corollary 1 In view of Lemma 3, our problem (1) has the following solution:

$$
z(t)=\left\{\begin{array}{l}
\left.\frac{1}{\Gamma(\rho)} \int_{0}^{t}(t-\xi)\right)^{\rho-1} \mathrm{~F}\left(\xi, z(\xi),{ }_{0}^{C} \mathrm{D}_{t_{i}}^{\rho} z(\xi)\right) d \xi+\mathcal{B}(1-t), \quad t \in \mathrm{J}_{0} ; \\
\frac{1}{\Gamma(\rho)} \int_{t_{k}}^{t}(t-\xi){ }^{\rho-1} \mathrm{~F}\left(\xi, z(\xi),{ }_{0}^{C} \mathrm{D}_{t_{i}}^{\rho} z(\xi)\right) d \xi \\
\left.\left.\quad+\frac{1}{\Gamma(\rho)} \sum_{i=1}^{k} \int_{t_{i-1}}^{t_{i}}\left(t_{i}-\xi\right)\right)^{\rho-1} \mathrm{~F}\left(\xi, z(\xi),{ }_{0}^{C} \mathrm{D}_{t_{i}}^{\rho} z(\xi)\right)\right) d \xi \\
\quad+\frac{1}{\Gamma(\rho-1)} \sum_{i=1}^{k}\left(t-t_{i}\right) \int_{t_{i-1}}^{t_{i}}\left(t_{i}-\xi\right)^{\rho-2} \mathrm{~F}\left(\xi, z(\xi),{ }_{0}^{C} \mathrm{D}_{t_{i}}^{\rho} z(\xi)\right) d \xi \\
\quad+\sum_{i=1}^{k}\left(t-t_{i}\right) \tilde{\mathrm{I}}_{i}\left(z\left(t_{i}\right)\right)+\sum_{i=1}^{k} \mathrm{I}_{i}\left(z\left(t_{i}\right)\right)+\mathcal{B}(1-t), \quad t \in \mathrm{J}_{k}, k=1,2,3, \ldots, m,
\end{array}\right.
$$

where

$$
\begin{aligned}
\mathcal{B}= & \left.\frac{1}{\Gamma(\rho)} \sum_{i=1}^{m+1} \int_{t_{i-1}}^{t_{i}}\left(t_{i}-\xi\right)^{\rho-1} \mathrm{~F}\left(\xi, z(\xi),{ }_{0}^{C} \mathrm{D}_{t_{i}}^{\rho} z(\xi)\right)\right) d \xi \\
& \left.+\frac{1}{\Gamma(\rho-1)} \sum_{i=1}^{m}\left(1-t_{i}\right) \int_{t_{i-1}}^{t_{i}}\left(t_{i}-\xi\right)^{\rho-2} \mathrm{~F}\left(\xi, z(\xi),{ }_{0}^{C} \mathrm{D}_{t_{i}}^{\rho} z(\xi)\right)\right) d \xi \\
& +\frac{1}{\Gamma(\rho-1)} \int_{t_{i}}^{\varrho}(\varrho-\xi)^{\rho-2} \mathrm{~F}\left(\xi, z(\xi),{ }_{0}^{C} \mathrm{D}_{t_{i}}^{\rho} z(\xi)\right) d \xi \\
& +\frac{1}{\Gamma(\rho-1)} \sum_{i=1}^{n} \int_{t_{i-1}}^{t_{i}}\left(t_{i}-\xi\right)^{\rho-2} \mathrm{~F}\left(\xi, z(\xi),{ }_{0}^{C} \mathrm{D}_{t_{i}}^{\rho} z(\xi)\right) d \xi \\
& +\sum_{i=1}^{m}\left(1-t_{i}\right) \tilde{\mathrm{I}}_{i}\left(z\left(t_{i}\right)\right)+\sum_{i=1}^{n} \tilde{\mathrm{I}}_{i}\left(z\left(t_{i}\right)\right)+\sum_{i=1}^{m} \mathrm{I}_{i}\left(z\left(t_{i}\right)\right) .
\end{aligned}
$$

We use the notation $\vartheta_{z}(t)=\mathrm{F}\left(t, z(t),{ }_{0}^{C} \mathrm{D}_{t_{i}}^{\rho} z(t)\right)$.

\section{Main results}

To transform our problem to a fixed point problem, we define the operator $\mathscr{T}: \mathrm{E} \rightarrow \mathrm{E}$ by

$$
\begin{aligned}
(\mathscr{T} z)(t)= & \frac{1}{\Gamma(\rho)} \int_{t_{i}}^{t}(t-\xi)^{\rho-1} \vartheta_{z}(\xi) d \xi+\frac{1}{\Gamma(\rho)} \sum_{0<t_{i}<t} \int_{t_{i-1}}^{t_{i}}\left(t_{i}-\xi\right)^{\rho-1} \vartheta_{z}(\xi) d \xi \\
& +\frac{1}{\Gamma(\rho-1)} \sum_{0<t_{i}<t} \int_{t_{i-1}}^{t_{i}}\left(t_{i}-\xi\right)^{\rho-2} \vartheta_{z}(\xi) d \xi+\sum_{0<t_{i}<t}\left(t-t_{i}\right) \tilde{\mathrm{I}}_{i}\left(z\left(t_{i}\right)\right)
\end{aligned}
$$




$$
\begin{aligned}
& +\sum_{0<t_{i}<t} \mathrm{I}_{i}\left(z\left(t_{i}\right)\right)+(1-t)\left[\frac{1}{\Gamma(\rho)} \sum_{i=1}^{m+1} \int_{t_{i-1}}^{t_{i}}\left(t_{i}-\xi\right)^{\rho-1} \vartheta_{z}(\xi) d \xi\right. \\
& +\frac{1}{\Gamma(\rho-1)} \sum_{i=1}^{m}\left(1-t_{i}\right) \int_{t_{i-1}}^{t_{i}}\left(t_{i}-\xi\right)^{\rho-2} \vartheta_{z}(\xi) d \xi \\
& +\frac{1}{\Gamma(\rho-1)} \int_{t_{i}}^{\varrho}(\varrho-\xi)^{\rho-2} \vartheta_{z}(\xi) d \xi+\frac{1}{\Gamma(\rho-1)} \sum_{i=1}^{m} \int_{t_{i-1}}^{t_{i}}\left(t_{i}-\xi\right)^{\rho-2} \vartheta_{z}(\xi) d \xi \\
& \left.+\sum_{i=1}^{m}\left(1-t_{i}\right) \tilde{\mathrm{I}}_{i}\left(z\left(t_{i}\right)\right)+\sum_{i=1}^{m} \tilde{\mathrm{I}}_{i}\left(z\left(t_{i}\right)\right)+\sum_{i=1}^{m} \mathrm{I}_{i}\left(z\left(t_{i}\right)\right)\right] .
\end{aligned}
$$

Obviously, the fixed points of $\mathscr{T}$ are the solutions of problem (1).

We assume the following hypotheses for $i=1,2, \ldots, m$.

$\left(\mathrm{H}_{1}\right)$ The nonlinear function $\mathrm{F}:[0,1] \times \mathrm{R}^{2} \rightarrow \mathrm{R}$ is continuous;

$\left(\mathrm{H}_{2}\right)$ let us have constants $K>0, L \in(0,1)$, which satisfy

$$
|\mathrm{F}(t, z, \bar{z})-\mathrm{F}(t, u, \bar{u})| \leq K|z(t)-u(t)|+L|\bar{z}(t)-\bar{u}(t)|
$$

$\left(\mathrm{H}_{3}\right)$ the relation $\left|\mathrm{I}_{i}\left(z\left(t_{i}\right)\right)-\mathrm{I}_{i}\left(\bar{z}\left(t_{i}\right)\right)\right| \leq b\left|z\left(t_{i}\right)-\bar{z}\left(t_{i}\right)\right|$, holds with $b>0$;

$\left(\mathrm{H}_{4}\right)$ the relation $\left|\tilde{\mathrm{I}}_{i}\left(z\left(t_{i}\right)\right)-\tilde{\mathrm{I}}_{i}\left(\bar{z}\left(t_{i}\right)\right)\right| \leq b^{*}\left|z\left(t_{i}\right)-\bar{z}\left(t_{i}\right)\right|$, holds with $b^{*}>0$;

$\left(\mathrm{H}_{5}\right)$ there exist functions $p, q, r \in C\left(\mathrm{~J}, \mathrm{R}^{+}\right)$, with

$$
\left|\mathrm{F}\left(t, z(t),{ }_{0}^{C} \mathrm{D}_{t_{i}}^{\rho} z(t)\right)\right| \leq p(t)+q(t)|z|+r(t)\left|{ }_{0}^{C} \mathrm{D}_{t_{i}}^{\rho} z(t)\right|, \quad \text { for } t \in \mathrm{J}, z \in \mathrm{E},
$$

such that $r^{*}=\sup _{t \in \mathrm{J}}|r(t)|<1$;

$\left(\mathrm{H}_{6}\right)$ under the continuity of $\mathrm{I}_{i}, \tilde{\mathrm{I}}_{i}: \mathrm{R} \rightarrow \mathrm{R}$ there exist some constants $M^{*}, N^{*}, F^{*}, G^{*}>0$, with $\left|\mathrm{I}_{i}(z)\right| \leq M^{*}|z|+N^{*}$ and $\left|\tilde{\mathrm{I}}_{i}(z)\right| \leq F^{*}|z|+G^{*}$, for each $z \in \mathrm{R}, i=1,2, \ldots, m$.

Theorem 3 If the hypotheses $\left(\mathrm{H}_{1}\right)-\left(\mathrm{H}_{6}\right)$ hold then the considered problem (1) has at least one solution.

Proof This proof consists of a number of steps:

Step 1: To show that $\mathscr{T}$ is continuous, take $\left\{z_{n}\right\}$ to be a sequence such that $z_{n} \rightarrow z \in \mathrm{E}$. Then, corresponding to every $t \in \mathrm{J}$, we take

$$
\begin{aligned}
\left|\left(\mathscr{T}_{n}\right)(t)-(\mathscr{T} z)(t)\right| \leq & \frac{1}{\Gamma(\rho)} \int_{t_{i}}^{t}(t-\xi)^{\rho-1}\left|\vartheta_{z, n}(\xi)-\vartheta_{z}(\xi)\right| d \xi \\
& +\frac{1}{\Gamma(\rho)} \sum_{0<t_{i}<t} \int_{t_{i-1}}^{t_{i}}\left(t_{i}-\xi\right)^{\rho-1}\left|\vartheta_{z, n}(\xi)-\vartheta_{z}(\xi)\right| d \xi \\
& +\frac{1}{\Gamma(\rho-1)} \sum_{0<t_{i}<t} \int_{t_{i-1}}^{t_{i}}\left(t_{i}-\xi\right)^{\rho-2}\left|\vartheta_{z, n}(\xi)-\vartheta_{z}(\xi)\right| d \xi \\
& +\sum_{0<t_{i}<t}\left(t-t_{i}\right)\left|\tilde{\mathrm{I}}_{i}\left(z_{n}\left(t_{i}\right)\right)-I^{*}\left(z\left(t_{i}\right)\right)\right|+\sum_{0<t_{i}<t}\left|\mathrm{I}_{i}\left(z_{n}\left(t_{i}\right)\right)-\mathrm{I}_{i}\left(z\left(t_{i}\right)\right)\right| \\
& +\frac{1}{\Gamma(\rho)} \sum_{i=1}^{m+1} \int_{t_{i-1}}^{t_{i}}\left(t_{i}-\xi\right)^{\rho-1}\left|\vartheta_{z, n}(\xi)-\vartheta_{z}(\xi)\right| d \xi
\end{aligned}
$$




$$
\begin{aligned}
& +\frac{1}{\Gamma(\rho-1)} \sum_{i=1}^{m}\left(1-t_{i}\right) \int_{t_{i-1}}^{t_{i}}\left(t_{i}-\xi\right)^{\rho-2}\left|\vartheta_{z, n}(\xi)-\vartheta_{z}(\xi)\right| d \xi \\
& +\frac{1}{\Gamma(\rho-1)} \int_{t_{i}}^{\varrho}(\varrho-\xi)^{\rho-2}\left|\vartheta_{z, n}(\xi)-\vartheta_{z}(\xi)\right| d \xi \\
& +\frac{1}{\Gamma(\rho-1)} \sum_{i=1}^{m} \int_{t_{i-1}}^{t_{i}}\left(t_{i}-\xi\right)^{\rho-2}\left|\vartheta_{z, n}(\xi)-\vartheta_{z}(\xi)\right| d \xi \\
& +\sum_{i=1}^{m}\left(1-t_{i}\right)\left|\tilde{\mathrm{I}}_{i}\left(z_{n}\left(t_{i}\right)\right)-\tilde{\mathrm{I}}_{i}\left(z\left(t_{i}\right)\right)\right|+\sum_{i=1}^{m}\left|\tilde{\mathrm{I}}_{i}\left(z_{n}\left(t_{i}\right)\right)-\tilde{\mathrm{I}}_{i}\left(z\left(t_{i}\right)\right)\right| \\
& +\sum_{i=1}^{m}\left|\mathrm{I}_{i}\left(z_{n}\left(t_{i}\right)\right)-\mathrm{I}_{i}\left(z\left(t_{i}\right)\right)\right|
\end{aligned}
$$

where $\vartheta_{z, n}, \vartheta_{z} \in C(\mathrm{~J}, \mathrm{R})$ satisfy the functional equations

$$
\vartheta_{z, n}(t)=\mathrm{F}\left(t, z_{n}(t), \vartheta_{z, n}(t)\right), \quad \vartheta_{z}(t)=\mathrm{F}\left(t, z(t), \vartheta_{z}(t)\right)
$$

respectively. By $\left(\mathrm{H}_{2}\right)$, we get

$$
\left|\vartheta_{z, n}(t)-\vartheta_{z}(t)\right| \leq \frac{K}{1-L}\left\|z_{n}-z\right\|_{P C}
$$

Here $z_{n} \rightarrow z$ as $n \rightarrow \infty$ implies $\vartheta_{z, n}(t) \rightarrow \vartheta_{z}(t)$ as $n \rightarrow \infty$, for each $t \in \mathrm{J}$. Also as every convergent sequence is bounded and we let there exist a real constant $\mathbb{k}>0$ such that, for each $t \in \mathrm{J}$, we have $\left|\vartheta_{z, n}(t)\right| \leq \mathbb{k}$ and $\left|\vartheta_{z}(t)\right| \leq \mathbb{k}$, then

$$
\begin{aligned}
(t-\xi)^{\rho-1}\left|\vartheta_{z, n}(\xi)-\vartheta_{z}(\xi)\right| & \leq(t-\xi)^{\rho-1}\left(\left|\vartheta_{z, n}(\xi)\right|+\left|\vartheta_{z}(\xi)\right|\right. \\
& \leq 2 \mathbb{k}(t-\xi)^{\rho-1} \\
\left(t_{i}-\xi\right)^{\rho-1}\left|\vartheta_{z, n}(\xi)-\vartheta_{z}(\xi)\right| & \leq\left(t_{i}-\xi\right)^{\rho-1}\left(\left|\vartheta_{z, n}(\xi)\right|+\left|\vartheta_{z}(\xi)\right|\right. \\
& \leq 2 \mathbb{k}\left(t_{i}-\xi\right)^{\rho-1} \\
(t-\xi)^{\rho-2}\left|\vartheta_{z, n}(\xi)-\vartheta_{z}(\xi)\right| & \leq(t-\xi)^{\rho-2}\left(\left|\vartheta_{z, n}(\xi)\right|+\left|\vartheta_{z}(\xi)\right|\right. \\
& \leq 2 \mathbb{k}(t-\xi)^{\rho-2}
\end{aligned}
$$

and

$$
\begin{aligned}
\left(t_{i}-\xi\right)^{\rho-2}\left|\vartheta_{z, n}(\xi)-\vartheta_{z}(\xi)\right| & \leq\left(t_{i}-\xi\right)^{\rho-2}\left(\left|\vartheta_{z, n}(\xi)\right|+\left|\vartheta_{z}(\xi)\right|\right. \\
& \leq 2 \mathbb{k}\left(t_{i}-\xi\right)^{\rho-2} .
\end{aligned}
$$

The functions $\xi \rightarrow 2 \mathbb{k}(t-\xi)^{\rho-1}, \xi \rightarrow 2 \mathbb{k}\left(t_{i}-\xi\right)^{\rho-1}, \xi \rightarrow 2 \mathbb{k}(t-\xi)^{\rho-2}$ and $\xi \rightarrow 2 \mathbb{k}\left(t_{i}-\xi\right)^{\rho-2}$ are integrable for each $t \in \mathrm{J}$ on $[0, t]$. Also since $\mathrm{F}, \mathrm{I}, \overline{\mathfrak{I}}$ are continuous, hence by Lebesgue dominated convergent theorem, from (11), we have

$$
\left|\mathscr{T} z_{n}(t)-\mathscr{T} z(t)\right| \rightarrow 0 \quad \text { as } n \rightarrow \infty
$$


Hence

$$
\left\|\mathscr{T} z_{n}-\mathscr{T} z\right\|_{P C} \rightarrow 0 \quad \text { as } n \rightarrow \infty
$$

Therefore, the operator $T$ is continuous.

Step 2: Next we show that $\mathscr{T}$ maps bounded sets into bounded sets. Indeed, we just need to show that, for any $\lambda>0$, there exists a constant $\varpi>0$ such that, for every $z \in \mathfrak{D}_{\lambda}=\{z \in$ $\left.\mathrm{E}:\|z\|_{P C} \leq \lambda\right\}$, one has $\|\mathscr{T} z\|_{P C} \leq \varpi$. For every $t \in \mathrm{J}$, we obtain

$$
\begin{aligned}
|(\mathscr{T} z)(t)| \leq & \frac{1}{\Gamma(\rho)} \int_{t_{i}}^{t}(t-\xi)^{\rho-1}\left|\vartheta_{z}(\xi)\right| d \xi+\frac{1}{\Gamma(\rho)} \sum_{0<t_{i}<t} \int_{t_{i-1}}^{t_{i}}\left(t_{i}-\xi\right)^{\rho-1}\left|\vartheta_{z}(\xi)\right| d \xi \\
& +\frac{1}{\Gamma(\rho-1)} \sum_{0<t_{i}<t}\left(t-t_{i}\right) \int_{t_{i-1}}^{t_{i}}\left(t_{i}-\xi\right)^{\rho-2}\left|\vartheta_{z}(\xi)\right| d \xi \\
& +\sum_{0<t_{i}<t}\left(t-t_{i}\right)\left|\tilde{\mathrm{I}}_{i}\left(z\left(t_{i}\right)\right)\right|+\sum_{0<t_{i}<t}\left|\mathrm{I}_{i}\left(z\left(t_{i}\right)\right)\right| \\
& +\frac{1}{\Gamma(\rho)} \sum_{i=1}^{m+1} \int_{t_{i-1}}^{t_{i}}\left(t_{i}-\xi\right)^{\rho-1}\left|\vartheta_{z}(\xi)\right| d \xi+\frac{1}{\Gamma(\rho-1)} \int_{t_{i}}^{\varrho}(\varrho-\xi)^{\rho-2}\left|\vartheta_{z}(\xi)\right| d \xi \\
& +\frac{1}{\Gamma(\rho-1)} \sum_{i=1}^{m}\left(1-t_{i}\right) \int_{t_{i-1}}^{t_{i}}\left(t_{i}-\xi\right)^{\rho-2}\left|\vartheta_{z}(\xi)\right| d \xi \\
& +\frac{1}{\Gamma(\rho-1)} \sum_{i=1}^{m} \int_{t_{i-1}}^{t_{i}}\left(t_{i}-\xi\right)^{\rho-2}\left|\vartheta_{z}(\xi)\right| d \xi \\
& +\sum_{i=1}^{m}\left(1-t_{i}\right)\left|\tilde{\mathrm{I}}_{i}\left(z\left(t_{i}\right)\right)\right|+\sum_{i=1}^{m}\left|\tilde{\mathrm{I}}_{i}\left(z\left(t_{i}\right)\right)\right|+\sum_{i=1}^{m}\left|\mathrm{I}_{i}\left(z\left(t_{i}\right)\right)\right|
\end{aligned}
$$

where $\vartheta_{z}$ is given in (12). Using $\left(\mathrm{H}_{5}\right)$, for each $t \in \mathrm{J}$ and using $p^{*}=\sup _{t \in \mathrm{J}}|p(t)|, q^{*}=$ $\sup _{t \in \mathrm{J}}|q(t)|$, we have

$$
\begin{aligned}
\left|\vartheta_{z}(t)\right| & =\left|\mathrm{F}\left(t, z(t), \vartheta_{z}(t)\right)\right| \leq p(t)+q(t)|z|+r(t)\left|\vartheta_{z}(t)\right| \\
& \leq p^{*}+q^{*} \lambda+r^{*}\left|\vartheta_{z}(t)\right|,
\end{aligned}
$$

which yields

$$
\left\|\vartheta_{z}\right\|_{P C} \leq \frac{1}{1-r^{*}}\left(p^{*}+q^{*} \lambda\right)=: \mu .
$$

Thanks to (15), the inequality (14) yields

$$
\begin{aligned}
|(\mathscr{T} z)(t)| \leq & \frac{\mu}{\Gamma(\rho)} \int_{t_{i}}^{t}(t-\xi)^{\rho-1} d \xi+\frac{2 \mu}{\Gamma(\rho)} \sum_{i=1}^{m+1} \int_{t_{i-1}}^{t_{i}}\left(t_{i}-\xi\right)^{\rho-1} d \xi \\
& +\frac{3 \mu}{\Gamma(\rho-1)} \sum_{i=1}^{m} \int_{t_{i-1}}^{t_{i}}\left(t_{i}-\xi\right)^{\rho-2} d \xi+\frac{\mu}{\Gamma(\rho-1)} \int_{t_{i}}^{\varrho}(\varrho-\xi)^{\rho-2} d \xi \\
& +2 m b+3 m b^{*} \\
\leq & \mu\left(\frac{2 m+3}{\Gamma(\rho+1)}+\frac{3 m+1}{\Gamma(\rho)}\right)+m\left(2 b+3 b^{*}\right),
\end{aligned}
$$


which further gives

$$
\|\mathscr{T} z\|_{P C} \leq \mu\left(\frac{2 m+3}{\Gamma(\rho+1)}+\frac{3 m+1}{\Gamma(\rho)}\right)+m\left(2 b+3 b^{*}\right)=\varpi .
$$

Step 3: To show that $\mathscr{T}$ is equi-continuous, let $\mathfrak{D}_{\lambda} \subseteq \mathrm{E}$, then, for $z \in \mathfrak{D}_{\lambda}$ and $t_{1}, t_{2} \in \mathrm{J}$ with $t_{1}<t_{2}$, we get

$$
\begin{aligned}
& \left|(\mathscr{T} z)\left(t_{2}\right)-(\mathscr{T} z)\left(t_{1}\right)\right| \\
& \leq\left|\frac{1}{\Gamma(\rho)} \int_{t_{i}}^{t_{2}}\left(t_{2}-\xi\right)^{\rho-1} \vartheta_{z}(\xi) d \xi-\frac{1}{\Gamma(\rho)} \int_{t_{i}}^{t_{1}}\left(t_{1}-\xi\right)^{\rho-1} \vartheta_{z}(\xi) d \xi\right| \\
& \quad+\frac{1}{\Gamma(\rho)} \sum_{\left.0<t_{i}<t_{2}-t_{1}\right)} \int_{t_{i-1}}^{t_{i}}\left(t_{i}-\xi\right)^{\rho-1}\left|\vartheta_{z}(\xi)\right| d \xi+\sum_{0<t_{i}<\left(t_{2}-t_{1}\right)}\left(t_{2}-t_{1}\right)\left|\tilde{\mathrm{I}}_{i}\left(z\left(t_{i}\right)\right)\right| \\
& \quad+\frac{1}{\Gamma(\rho-1)} \sum_{0<t_{i}<\left(t_{2}-t_{1}\right)}\left(t_{2}-t_{1}\right) \int_{t_{i-1}}^{t_{i}}\left(t_{i}-\xi\right)^{\rho-2}\left|\vartheta_{z}(\xi)\right| d \xi+\sum_{0<t_{i}<\left(t_{2}-t_{1}\right)}\left|\mathrm{I}_{i}\left(z\left(t_{i}\right)\right)\right| \\
& \leq \mu\left(\frac{1}{\Gamma(\rho+1)}-\frac{1}{\Gamma(\rho+1)}\right)+\frac{\mu\left(t_{2}-t_{1}\right)}{\Gamma(\rho+1)}+\left(t_{2}-t_{1}\right)\left(t_{2}-t_{1}\right)\left(F^{*}|z|+G^{*}\right) \\
& \quad+\frac{\mu\left(t_{2}-t_{1}\right)}{\Gamma(\rho)}\left(t_{2}-t_{1}\right)+\left(t_{2}-t_{1}\right)\left(M^{*}|z|+N^{*}\right) \rightarrow 0 \quad \text { as } t_{1} \rightarrow t_{2} .
\end{aligned}
$$

Hence

$$
\left|(\mathscr{T} z)\left(t_{2}\right)-(\mathscr{T} z)\left(t_{1}\right)\right| \rightarrow 0, \quad \text { as } t_{1} \rightarrow t_{2} .
$$

Thanks to Ascoli-Arzelà theorem, the operator $\mathscr{T}: \mathrm{E} \rightarrow \mathrm{E}$ is completely continuous.

Step 4: Finally, we show that the set $\mathrm{W}=\{z \in \mathrm{E}: z=\eta \mathscr{T} z$, for some $0<\eta<1\}$ is bounded, such that, for $z \in \mathrm{W}$, and $z=\eta \mathscr{T} z$, with $0<\eta<1$, hold. Then for every $t \in \mathrm{J}$, we take

$$
\begin{aligned}
z(t)= & \frac{\eta}{\Gamma(\rho)} \int_{t_{i}}^{t}(t-\xi)^{\rho-1} \vartheta_{z}(\xi) d \xi+\frac{\eta}{\Gamma(\rho)} \sum_{0<t_{i}<t} \int_{t_{i-1}}^{t_{i}}\left(t_{i}-\xi\right)^{\rho-1} \vartheta_{z}(\xi) d \xi \\
& +\frac{\eta}{\Gamma(\rho-1)} \sum_{0<t_{i}<t}\left(t-t_{i}\right) \int_{t_{i-1}}^{t_{i}}\left(t_{i}-\xi\right)^{\rho-2} \vartheta_{z}(\xi) d \xi+\eta \sum_{0<t_{i}<t}\left(t-t_{i}\right)\left|\tilde{\mathrm{I}}_{i}\left(z\left(t_{i}\right)\right)\right| \\
& +\eta \sum_{0<t_{i}<t}\left|\mathrm{I}_{i}\left(z\left(t_{i}\right)\right)\right|+\frac{\eta}{\Gamma(\rho)} \sum_{i=1}^{m+1} \int_{t_{i-1}}^{t_{i}}\left(t_{i}-\xi\right)^{\rho-1} \vartheta_{z}(\xi) d \xi \\
& +\frac{\eta}{\Gamma(\rho-1)} \int_{t_{i}}^{\varrho}(\varrho-\xi)^{\rho-2} \vartheta_{z}(\xi) d \xi \\
& +\frac{\eta}{\Gamma(\rho-1)} \sum_{i=1}^{m}\left(1-t_{i}\right) \int_{t_{i-1}}^{t_{i}}\left(t_{i}-\xi\right)^{\rho-2} \vartheta_{z}(\xi) d \xi \\
& +\frac{\eta}{\Gamma(\rho-1)} \sum_{i=1}^{m} \int_{t_{i-1}}^{t_{i}}\left(t_{i}-\xi\right)^{\rho-2} \vartheta_{z}(\xi) d \xi \\
& +\eta \sum_{i=1}^{m}\left(1-t_{i}\right)\left|\tilde{\mathrm{I}}_{i}\left(z\left(t_{i}\right)\right)\right|+\eta \sum_{i=1}^{m}\left|\tilde{\mathrm{I}}_{i}\left(z\left(t_{i}\right)\right)\right|+\eta \sum_{i=1}^{m}\left|\mathrm{I}_{i}\left(z\left(t_{i}\right)\right)\right| .
\end{aligned}
$$


By using (15) and $0<\eta<1(16)$ implies that

$$
\begin{aligned}
& |z(t)| \leq \frac{1}{\Gamma(\rho)} \int_{t_{i}}^{t}(t-\xi)^{\rho-1}\left|\vartheta_{z}(\xi)\right| d \xi \\
& +\frac{1}{\Gamma(\rho)} \sum_{0<t_{i}<t} \int_{t_{i-1}}^{t_{i}}\left(t_{i}-\xi\right)^{\rho-1}\left|\vartheta_{z}(\xi)\right| d \xi \\
& +\frac{1}{\Gamma(\rho-1)} \sum_{0<t_{i}<t}\left(t-t_{i}\right) \int_{t_{i-1}}^{t_{i}}\left(t_{i}-\xi\right)^{\rho-2}\left|\vartheta_{z}(\xi)\right| d \xi+\sum_{0<t_{i}<t}\left(t-t_{i}\right)\left|\tilde{\mathrm{I}}_{i}\left(z\left(t_{i}\right)\right)\right| \\
& +\sum_{0<t_{i}<t}\left|\mathrm{I}_{i}\left(z\left(t_{i}\right)\right)\right|+\frac{1}{\Gamma(\rho)} \sum_{i=1}^{m+1} \int_{t_{i-1}}^{t_{i}}\left(t_{i}-\xi\right)^{\rho-1}\left|\vartheta_{z}(\xi)\right| d \xi \\
& +\frac{1}{\Gamma(\rho-1)} \int_{t_{i}}^{\varrho}(\varrho-\xi)^{\rho-2}\left|\vartheta_{z}(\xi)\right| d \xi \\
& +\frac{1}{\Gamma(\rho-1)} \sum_{i=1}^{m}\left(1-t_{i}\right) \int_{t_{i-1}}^{t_{i}}\left(t_{i}-\xi\right)^{\rho-2}\left|\vartheta_{z}(\xi)\right| d \xi \\
& +\frac{1}{\Gamma(\rho-1)} \sum_{i=1}^{n} \int_{t_{i-1}}^{t_{i}}\left(t_{i}-\xi\right)^{\rho-2}\left|\vartheta_{z}(\xi)\right| d \xi \\
& +\sum_{i=1}^{m}\left(1-t_{i}\right)\left|\tilde{\mathrm{I}}_{i}\left(z\left(t_{i}\right)\right)\right|+\sum_{i=1}^{m}\left|\tilde{\mathrm{I}}_{i}\left(z\left(t_{i}\right)\right)\right|+\sum_{i=1}^{m}\left|\mathrm{I}_{i}\left(z\left(t_{i}\right)\right)\right| \\
& \leq \frac{\mu}{\Gamma(\rho)} \int_{t_{i}}^{t}(t-\xi)^{\rho-1} d \xi+\frac{2 \mu}{\Gamma(\rho)} \sum_{i=1}^{m+1} \int_{t_{i-1}}^{t_{i}}\left(t_{i}-\xi\right)^{\rho-1} d \xi \\
& +\frac{3 \mu}{\Gamma(\rho-1)} \sum_{i=1}^{m}\left(1-t_{i}\right) \int_{t_{i-1}}^{t_{i}}\left(t_{i}-\xi\right)^{\rho-2} d \xi \\
& +\frac{\mu}{\Gamma(\rho-1)} \int_{t_{i}}^{\varrho}(\varrho-\xi)^{\rho-2} d \xi+2 m b+3 m b^{*} \\
& \leq \mu\left(\frac{2 m+3}{\Gamma(\rho+1)}+\frac{3 m+1}{\Gamma(\rho)}\right)+m\left(2 b+3 b^{*}\right) \text {. }
\end{aligned}
$$

This further gives

$$
\|z\|_{P C} \leq \mu\left(\frac{2 m+3}{\Gamma(\rho+1)}+\frac{3 m+1}{\Gamma(\rho)}\right)+m\left(2 b+3 b^{*}\right) .
$$

Thus, we conclude that the set W is bounded. Hence as a consequence of Schaefer's fixed point theorem $\mathscr{T}$ has at least one fixed point which is the solution of problem (1).

Theorem 4 The boundary value problem (BVP) (1) has a unique solution under the hypotheses $\left(\mathrm{H}_{1}\right)-\left(\mathrm{H}_{4}\right)$ and the inequality

$$
\Upsilon=\left[\frac{K}{1-L}\left(\frac{2 m+3}{\Gamma(\rho+1)}+\frac{3 m+1}{\Gamma(\rho)}\right)+m\left(2 b+3 b^{*}\right)\right]<1 .
$$


Proof Let $z, \bar{z} \in \mathrm{E}$ and $t \in \mathrm{J}$, then one has

$$
\begin{aligned}
|(\mathscr{T} z)(t)-(\mathscr{T} \bar{z})(t)| \leq & \frac{1}{\Gamma(\rho)} \int_{t_{i}}^{t}(t-\xi)^{\rho-1}\left|\vartheta_{z}(\xi)-\delta_{\bar{z}}(\xi)\right| d \xi \\
& +\frac{1}{\Gamma(\rho)} \sum_{0<t_{i}<t} \int_{t_{i-1}}^{t_{i}}\left(t_{i}-\xi\right)^{\rho-1}\left|\vartheta_{z}(\xi)-\delta_{\bar{z}}(\xi)\right| d \xi \\
& +\frac{1}{\Gamma(\rho-1)} \sum_{0<t_{i}<t} \int_{t_{i-1}}^{t_{i}}\left(t_{i}-\xi\right)^{\rho-2}\left|\vartheta_{z}(\xi)-\delta_{\bar{z}}(\xi)\right| d \xi \\
& +\sum_{0<t_{i}<t}\left(t-t_{i}\right)\left|\tilde{\mathrm{I}}_{i}\left(z\left(t_{i}\right)\right)-I^{*}\left(\bar{z}\left(t_{i}\right)\right)\right|+\sum_{0<t_{i}<t}\left|\mathrm{I}_{i}\left(z\left(t_{i}\right)\right)-\mathrm{I}_{i}\left(\bar{z}\left(t_{i}\right)\right)\right| \\
& +\frac{1}{\Gamma(\rho)} \sum_{i=1}^{m+1} \int_{t_{i-1}}^{t_{i}}\left(t_{i}-\xi\right)^{\rho-1}\left|\vartheta_{z}(\xi)-\delta_{\bar{z}}(\xi)\right| d \xi \\
& +\frac{1}{\Gamma(\rho-1)} \sum_{i=1}^{m}\left(1-t_{i}\right) \int_{t_{i-1}}^{t_{i}}\left(t_{i}-\xi\right)^{\rho-2}\left|\vartheta_{z}(\xi)-\delta_{\bar{z}}(\xi)\right| d \xi \\
& +\frac{1}{\Gamma(\rho-1)} \int_{t_{i}}^{\varrho}(\varrho-\xi)^{\rho-2}\left|\vartheta_{z}(\xi)-\delta_{\bar{z}}(\xi)\right| d \xi \\
& +\frac{1}{\Gamma(\rho-1)} \sum_{i=1}^{m} \int_{t_{i-1}}^{t_{i}}\left(t_{i}-\xi\right)^{\rho-2}\left|\vartheta_{z}(\xi)-\delta_{\bar{z}}(\xi)\right| d \xi \\
& +\sum_{i=1}^{m}\left(1-t_{i}\right)\left|\tilde{\mathrm{I}}_{i}\left(z\left(t_{i}\right)\right)-\tilde{\mathrm{I}}_{i}\left(\bar{z}\left(t_{i}\right)\right)\right|+\sum_{i=1}^{m}\left|\tilde{\mathrm{I}}_{i}\left(z\left(t_{i}\right)\right)-\tilde{\mathrm{I}}_{i}\left(\bar{z}\left(t_{i}\right)\right)\right| \\
& +\sum_{i=1}^{m}\left|\mathrm{I}_{i}\left(z\left(t_{i}\right)\right)-\mathrm{I}_{i}\left(\bar{z}\left(t_{i}\right)\right)\right|, \\
&
\end{aligned}
$$

where

$$
\vartheta_{z}(t)=\mathrm{F}\left(t, z(t), \vartheta_{z}(t)\right), \quad \delta_{\bar{z}}(t)=\mathrm{F}\left(t, \bar{z}(t), \delta_{\bar{z}}(t)\right) .
$$

With the use of $\left(\mathrm{H}_{2}\right)$, one has

$$
\left\|\vartheta_{z}-\delta_{\bar{z}}\right\|_{P C} \leq \frac{K}{1-L}\|z-\bar{z}\|_{P C}
$$

Therefore, using (20) in (18), we obtain

$$
\begin{aligned}
\|\mathscr{T} z-\mathscr{T}\|_{P C} \leq & \frac{K\|z-\bar{z}\|_{P C}}{\Gamma(\rho)(1-L)} \int_{t_{i}}^{t}(t-\xi)^{\rho-1} d \xi+\frac{2 K\|z-\bar{z}\|_{P C}}{\Gamma(\rho)(1-L)} \sum_{i=1}^{m+1} \int_{t_{i-1}}^{t_{i}}\left(t_{i}-\xi\right)^{\rho-1} d \xi \\
& +\frac{3 K\|z-\bar{z}\|_{P C}}{\Gamma(\rho-1)(1-L)} \sum_{i=1}^{m} \int_{t_{i-1}}^{t_{i}}\left(t_{i}-\xi\right)^{\rho-2} d \xi \\
& +\frac{K\|z-\bar{z}\|_{P C}}{\Gamma(\rho-1)(1-L)} \int_{t_{i}}^{\varrho}(\varrho-\xi)^{\rho-2} d \xi \\
& +2 m b\|z-\bar{z}\|_{P C}+3 m b^{*}\|z-\bar{z}\|_{P C} \\
\leq & \Upsilon\|z-\bar{z}\|_{P C} .
\end{aligned}
$$


Therefore, we get

$$
\|\mathscr{T} z-\mathscr{T} \bar{z}\|_{P C} \leq \Upsilon\|z-\bar{z}\|_{P C}
$$

Thus in view of Banach's contraction principle, problem (1) has a unique solution.

\section{Ulam-Hyers stability analysis}

In this section we investigate results concerning the Hyers-Ulam stability to the problem (1).

Theorem 5 If the hypotheses $\left(\mathrm{H}_{1}\right)-\left(\mathrm{H}_{4}\right)$ together with the inequality (17) are satisfied then the proposed problem (1) is Hyers-Ulam stable and generalized Hyers-Ulam stable.

Proof Corresponding to any solution $\bar{z} \in E$ of the inequality (2) let $z \in E$ be the unique solution to the given problem

$$
\left\{\begin{array}{l}
{ }_{0}^{C} \mathrm{D}_{t_{i}}^{\rho} z(t)=\vartheta_{z}(t), \quad 0<t<1, t \neq t_{i}, i=1,2, \ldots, m, \\
\left.\Delta z(t)\right|_{t=t_{i}}=\mathrm{I}_{i}\left(z\left(t_{i}\right)\right),\left.\quad \Delta z^{\prime}(t)\right|_{t=t_{i}}=\tilde{\mathrm{I}}_{i}\left(z\left(t_{i}\right)\right), \quad i=1,2, \ldots, m, \\
\left.z(t)\right|_{t=0}=-\left.z^{\prime}(t)\right|_{t=0},\left.\quad z(t)\right|_{t=1}=-z^{\prime}(t)_{t=\varrho}, \\
\quad \varrho \in(0,1), \varrho \neq t_{i} \text { for } i=, 1,2, \ldots, m, .
\end{array}\right.
$$

Then, inview of Lemma 3, we have

$$
\begin{aligned}
z(t)= & \frac{1}{\Gamma(\rho)} \int_{t_{i}}^{t}(t-\xi)^{\rho-1} \vartheta_{z}(\xi) d \xi+\frac{1}{\Gamma(\rho)} \sum_{i=1}^{i} \int_{t_{i-1}}^{t_{i}}\left(t_{i}-\xi\right)^{\rho-1} \vartheta_{z}(\xi) d \xi \\
& +\frac{1}{\Gamma(\rho-1)} \sum_{i=1}^{i} \int_{t_{i-1}}^{t_{i}}\left(t_{i}-\xi\right)^{\rho-2} \vartheta_{z}(\xi) d \xi+\sum_{i=1}^{i}\left(t-t_{i}\right) \tilde{\mathrm{I}}_{i}\left(z\left(t_{i}\right)\right)+\sum_{i=1}^{m} \mathrm{I}_{i}\left(z\left(t_{i}\right)\right) \\
& +(1-t)\left[\frac{1}{\Gamma(\rho)} \sum_{i=1}^{m+1} \int_{t_{i-1}}^{t_{i}}\left(t_{i}-\xi\right)^{\rho-1} \vartheta_{z}(\xi) d \xi\right. \\
& +\frac{1}{\Gamma(\rho-1)} \sum_{i=1}^{m}\left(1-t_{i}\right) \int_{t_{i-1}}^{t_{i}}\left(t_{i}-\xi\right)^{\rho-2} \vartheta_{z}(\xi) d \xi \\
& +\frac{1}{\Gamma(\rho-1)} \int_{t_{i}}^{\varrho}(\varrho-\xi)^{\rho-2} \vartheta_{z}(\xi) d \xi+\frac{1}{\Gamma(\rho-1)} \sum_{i=1}^{n} \int_{t_{i-1}}^{t_{i}}\left(t_{i}-\xi\right)^{\rho-2} \vartheta_{z}(\xi) d \xi \\
& \left.+\sum_{i=1}^{m}\left(1-t_{i}\right) \tilde{\mathrm{I}}_{i}\left(z\left(t_{i}\right)\right)+\sum_{i=1}^{n} \tilde{\mathrm{I}}_{i}\left(z\left(t_{i}\right)\right)+\sum_{i=1}^{m} \mathrm{I}_{i}\left(z\left(t_{i}\right)\right)\right] .
\end{aligned}
$$

Further if $\bar{z}$ is the solution of inequality (2) and using Remark 1, we get

$$
\left\{\begin{array}{l}
{ }_{0}^{C} \mathrm{D}_{t_{i}}^{\rho} \bar{z}(t)=\mathrm{F}\left(t, \bar{z}(t),{ }_{0}^{C} \mathrm{D}_{t_{i}}^{\rho} \bar{z}(t)\right)+\phi(t), \quad t \in \mathrm{J}_{i}, i=1,2, \ldots, m, \\
\left.\Delta \bar{z}(t)\right|_{t=t_{i}}=\mathrm{I}_{k}\left(\bar{z}\left(t_{i}\right)\right)+\phi_{i}, \quad i=1,2, \ldots, m, \\
\left.\Delta \bar{z}^{\prime}(t)\right|_{t=t_{i}}=\tilde{\mathrm{I}}\left(\bar{z}\left(t_{i}\right)\right)+\phi_{i}, \quad i=1,2, \ldots, m .
\end{array}\right.
$$


The solution of (21) is

$$
\begin{aligned}
& \bar{z}(t)=\frac{1}{\Gamma(\rho)} \int_{t_{i}}^{t}(t-\xi)^{\rho-1} \delta_{\bar{z}}(\xi) d \xi+\frac{1}{\Gamma(\rho)} \int_{t_{i}}^{t}(t-\xi)^{\rho-1} \phi(\xi) d \xi \\
& +\frac{1}{\Gamma(\rho)} \sum_{i=1}^{m} \int_{t_{i-1}}^{t_{i}}\left(t_{i}-\xi\right)^{\rho-1} \delta_{\bar{z}}(\xi) d \xi+\frac{1}{\Gamma(\rho)} \sum_{i=1}^{m} \int_{t_{i-1}}^{t_{i}}\left(t_{i}-\xi\right)^{\rho-1} \phi(\xi) d \xi \\
& +\frac{1}{\Gamma(\rho-1)} \sum_{i=1}^{m} \int_{t_{i-1}}^{t_{i}}\left(t_{i}-\xi\right)^{\rho-2} \delta_{\bar{z}}(\xi) d \xi+\frac{1}{\Gamma(\rho-1)} \sum_{i=1}^{m} \int_{t_{i-1}}^{t_{i}}\left(t_{i}-\xi\right)^{\rho-2} \phi(\xi) d \xi \\
& +\sum_{i=1}^{m}\left(t-t_{i}\right) \tilde{\mathrm{I}}_{i}\left(\bar{z}\left(t_{i}\right)\right)+\sum_{i=1}^{m}\left(t-t_{i}\right) \phi_{i}+\sum_{i=1}^{m} \mathrm{I}_{i}\left(\bar{z}\left(t_{i}\right)\right)+\sum_{i=1}^{m} \phi_{i} \\
& +(1-t)\left[\frac{1}{\Gamma(\rho)} \sum_{i=1}^{m+1} \int_{t_{i-1}}^{t_{i}}\left(t_{i}-\xi\right)^{\rho-1} \delta(\xi) d \xi+\frac{1}{\Gamma(\rho)} \sum_{i=1}^{m+1} \int_{t_{i-1}}^{t_{i}}\left(t_{i}-\xi\right)^{\rho-1} \phi(\xi) d \xi\right. \\
& +\frac{1}{\Gamma(\rho-1)} \sum_{i=1}^{m}\left(1-t_{i}\right) \int_{t_{i-1}}^{t_{i}}\left(t_{i}-\xi\right)^{\rho-2} \delta_{\bar{z}}(\xi) d \xi \\
& +\frac{1}{\Gamma(\rho-1)} \sum_{i=1}^{m}\left(1-t_{i}\right) \int_{t_{i-1}}^{t_{i}}\left(t_{i}-\xi\right)^{\rho-2} \phi(\xi) d \xi \\
& +\frac{1}{\Gamma(\rho-1)} \int_{t_{i}}^{\varrho}(\varrho-\xi)^{\rho-2} \delta(\xi) d \xi+\frac{1}{\Gamma(\rho-1)} \int_{t_{i}}^{\varrho}(\varrho-\xi)^{\rho-2} \phi(\xi) d \xi \\
& +\frac{1}{\Gamma(\rho-1)} \sum_{i=1}^{m} \int_{t_{i-1}}^{t_{i}}\left(t_{i}-\xi\right)^{\rho-2} \delta_{\bar{z}}(\xi) d \xi+\frac{1}{\Gamma(\rho-1)} \sum_{i=1}^{m} \int_{t_{i-1}}^{t_{i}}\left(t_{i}-\xi\right)^{\rho-2} \phi(\xi) d \xi \\
& +\sum_{i=1}^{m}\left(1-t_{i}\right) \tilde{\mathrm{I}}_{i}\left(\bar{z}\left(t_{i}\right)\right)+\sum_{i=1}^{m}\left(1-t_{i}\right) \phi_{i}+\sum_{i=1}^{m} \tilde{\mathrm{I}}_{i}\left(\bar{z}\left(t_{i}\right)\right)+\sum_{i=1}^{m} \phi_{i} \\
& \left.+\sum_{i=1}^{m} \mathrm{I}_{i}\left(\bar{z}\left(t_{i}\right)\right)+\sum_{i=1}^{m} \phi_{i}\right], \quad t \in \mathrm{J}_{i}
\end{aligned}
$$

Hence, for every $t \in \mathrm{J}_{i}$, one has

$$
\begin{aligned}
|\bar{z}(t)-z(t)| \leq & \frac{1}{\Gamma(\rho)} \int_{t_{i}}^{t}(t-\xi)^{\rho-1}\left|\delta_{\bar{z}}(\xi)-\vartheta_{z}(\xi)\right| d \xi+\frac{1}{\Gamma(\rho)} \int_{t_{i}}^{t}(t-\xi)^{\rho-1}|\phi(\xi)| d \xi \\
& +\frac{1}{\Gamma(\rho)} \sum_{i=1}^{m} \int_{t_{i-1}}^{t_{i}}\left(t_{i}-\xi\right)^{\rho-1}\left|\delta_{\bar{z}}(\xi)-\vartheta_{z}(\xi)\right| d \xi \\
& +\frac{1}{\Gamma(\rho)} \sum_{i=1}^{m} \int_{t_{i-1}}^{t_{i}}\left(t_{i}-\xi\right)^{\rho-1}|\phi(\xi)| d \xi \\
& +\frac{1}{\Gamma(\rho-1)} \sum_{i=1}^{m} \int_{t_{i-1}}^{t_{i}}\left(t_{i}-\xi\right)^{\rho-2}\left|\delta_{\bar{z}}(\xi)-\vartheta_{z}(\xi)\right| d \xi \\
& +\frac{1}{\Gamma(\rho-1)} \sum_{i=1}^{m} \int_{t_{i-1}}^{t_{i}}\left(t_{i}-\xi\right)^{\rho-2}|\phi(\xi)| d \xi \\
& +\sum_{i=1}^{m}\left(t-t_{i}\right)\left|\tilde{\mathrm{I}}_{i}\left(\bar{z}_{(}\left(t_{i}\right)\right)-\tilde{\mathrm{I}}_{i}\left(z\left(t_{i}\right)\right)\right|+\sum_{i=1}^{m}\left(t-t_{i}\right)\left|\phi_{i}\right|
\end{aligned}
$$




$$
\begin{aligned}
& +\sum_{i=1}^{m}\left|\mathrm{I}_{i}\left(\bar{z}\left(t_{i}\right)\right)-\mathrm{I}_{i}\left(z\left(t_{i}\right)\right)\right|+\sum_{i=1}^{m}\left|\phi_{i}\right| \\
& +\frac{1}{\Gamma(\rho)} \sum_{i=1}^{m+1} \int_{t_{i-1}}^{t_{i}}\left(t_{i}-\xi\right)^{\rho-1}\left|\delta_{\bar{z}}(\xi)-\vartheta_{z}(\xi)\right| d \xi \\
& +\frac{1}{\Gamma(\rho)} \sum_{i=1}^{m+1} \int_{t_{i-1}}^{t_{i}}\left(t_{i}-\xi\right)^{\rho-1}|\phi(\xi)| d \xi \\
& +\sum_{i=1}^{m}\left(1-t_{i}\right) \int_{t_{i-1}}^{t_{i}} \frac{\left(t_{i}-\xi\right)^{\rho-2}\left|\delta_{\bar{z}}(\xi)-\vartheta_{z}(\xi)\right|}{\Gamma(\rho-1)} d \xi \\
& +\int_{t_{i}}^{\varrho} \frac{(\varrho-\xi)^{\rho-2}\left|\delta_{\bar{z}}(\xi)-\vartheta_{z}(\xi)\right|}{\Gamma(\rho-1)} d \xi \\
& +\frac{1}{\Gamma(\rho-1)} \sum_{i=1}^{m}\left(1-t_{i}\right) \int_{t_{i-1}}^{t_{i}}\left(t_{i}-\xi\right)^{\rho-2}|\phi(\xi)| d \xi \\
& +\frac{1}{\Gamma(\rho-1)} \int_{t_{i}}^{\varrho}(\varrho-\xi)^{\rho-2}|\phi(\xi)| d \xi \\
& +\frac{1}{\Gamma(\rho-1)} \sum_{i=1}^{m} \int_{t_{i-1}}^{t_{i}}\left(t_{i}-\xi\right)^{\rho-2}\left|\delta_{\bar{z}}(\xi)-\vartheta_{z}(\xi)\right| d \xi \\
& +\frac{1}{\Gamma(\rho-1)} \sum_{i=1}^{m} \int_{t_{i-1}}^{t_{i}}\left(t_{i}-\xi\right)^{\rho-2}|\phi(\xi)| d \xi \\
& +\sum_{i=1}^{m}\left(1-t_{i}\right)\left|\tilde{\mathrm{I}}_{i}\left(\bar{z}\left(t_{i}\right)\right)-\tilde{\mathrm{I}}_{i}\left(\bar{z}\left(t_{i}\right)\right)-\tilde{\mathrm{I}}_{i}\left(z\left(t_{i}\right)\right)\right|+\sum_{i=1}^{m}\left(1-t_{i}\right)\left|+\sum_{i=1}^{m}\right| \phi_{i}\left|+\sum_{i=1}^{m}\right| \mathrm{I}_{i}\left(\bar{z}\left(t_{i}\right)\right)-\mathrm{I}_{i}\left(z\left(t_{i}\right)\right)\left|+\sum_{i=1}^{m}\right| \phi_{i} \mid .
\end{aligned}
$$

Hence by $\left(\mathrm{H}_{1}\right)-\left(\mathrm{H}_{4}\right)$ and using (20) along with (i) of Remark 1, one has

$$
\begin{aligned}
|\bar{z}(t)-z(t)| \leq & \frac{L\|\bar{z}-z\|_{P C}}{\Gamma(\rho)(1-L)} \int_{t_{i}}^{t}(t-\xi)^{\rho-1} d \xi+\frac{2 L\|\bar{z}-z\|_{P C}}{\Gamma(\rho)(1-L)} \sum_{i=1}^{m+1} \int_{t_{i-1}}^{t_{i}}\left(t_{i}-\xi\right)^{\rho-1} d \xi \\
& +\frac{3 L\|\bar{z}-z\|_{P C}}{\Gamma(\rho-1)(1-L)} \sum_{i=1}^{m} \int_{t_{i-1}}^{t_{i}}\left(t_{i}-\xi\right)^{\rho-2} d \xi \\
& +\frac{L\|\bar{z}-z\|_{P C}}{\Gamma(\rho-1)(1-L)} \int_{t_{i}}^{\varrho}(\varrho-\xi)^{\rho-2} d \xi \\
& +2 m b\|\bar{z}-z\|_{P C}+3 m b^{*}\|\bar{z}-z\|_{P C}+\frac{\epsilon}{\Gamma(\rho)} \int_{t_{i}}^{t}(t-\xi)^{\rho-1} d \xi \\
& +\frac{2 \epsilon}{\Gamma(\rho)} \sum_{i=1}^{m+1} \int_{t_{i-1}}^{t_{i}}\left(t_{i}-\xi\right)^{\rho-1} d \xi \\
& +\frac{3 \epsilon}{\Gamma(\rho-1)} \sum_{i=1}^{m} \int_{t_{i-1}}^{t_{i}}\left(t_{i}-\xi\right)^{\rho-2} d \xi+\frac{\epsilon}{\Gamma(\rho-1)} \int_{t_{i}}^{\varrho}(\varrho-\xi)^{\rho-2} d \xi+5 m \epsilon \\
\leq & \Upsilon\|\bar{z}-z\|_{P C}+\left[\frac{(2 m+3)}{\Gamma(\rho+1)}+\frac{3 m+1}{\Gamma(\rho)}+5 m\right] \epsilon .
\end{aligned}
$$


This yields

$$
\|\bar{z}-z\|_{P C} \leq \frac{\left[\frac{(2 m+3)}{\Gamma(\rho+1)}+\frac{3 m+1}{\Gamma(\rho)}+5 m\right] \epsilon}{1-\Upsilon} .
$$

Hence we have

$$
\|\bar{z}-z\|_{P C} \leq \mathbf{C}_{m, \rho} \epsilon,
$$

where

$$
\mathbf{C}_{m, \rho}=\frac{\left[\frac{(2 m+3)}{\Gamma(\rho+1)}+\frac{3 m+1}{\Gamma(\rho)}+5 m\right]}{1-\Upsilon} .
$$

Thus the solution of (1) is HU stable. Also by setting $\psi(\epsilon)=\mathbf{C}_{m, \rho} \epsilon ; \psi(0)=0$, the solution of (1) becomes GHU stable.

Assume that:

$\left(\mathrm{H}_{8}\right)$ For a nondecreasing function $\theta \in C(\mathrm{~J}, \mathrm{R})$, there exists $\beta_{\theta}>0$, such that, for any $t \in \mathrm{J}$

$$
I^{\rho} \theta(t) \leq \beta_{\theta} \theta(t) ; \quad \text { consequently } \quad I^{\rho-1} \theta(t) \leq \beta_{\theta} \theta(t) .
$$

Theorem 6 If the hypotheses $\left(\mathrm{H}_{1}\right)-\left(\mathrm{H}_{4}\right),\left(\mathrm{H}_{8}\right)$ and the inequality (17) are satisfied, then problem (1) is HUR stable with respect to $(\theta, \varphi)$ and consequently GHUR stable.

Proof We address for any solution $\bar{z} \in \mathrm{E}$ of inequality (4) and for unique solution $z$ the given problem

$$
\left\{\begin{array}{l}
{ }_{0}^{C} \mathrm{D}_{t_{i}}^{\rho} z(t)=\vartheta_{z}(t), \quad 0<t<1, t \neq t_{i}, i=1,2, \ldots, m, \\
\left.\Delta z(t)\right|_{t=t_{i}}=\mathrm{I}_{i}\left(z\left(t_{i}\right)\right),\left.\quad \Delta z^{\prime}(t)\right|_{t=t_{i}}=\tilde{\mathrm{I}}_{i}\left(z\left(t_{i}\right)\right), \quad i=1,2, \ldots, m, \\
\left.z(t)\right|_{t=0}=-\left.z^{\prime}(t)\right|_{t=0},\left.\quad z(t)\right|_{t=1}=-z^{\prime}(t) t=\varrho \\
\varrho \in(0,1), \varrho \neq t_{i} \text { for } i=, 1,2, \ldots, m .
\end{array}\right.
$$

From the proof of Theorem 5, we get

$$
\begin{aligned}
|\bar{z}(t)-z(t)| \leq & \frac{1}{\Gamma(\rho)} \int_{t_{i}}^{t}(t-\xi)^{\rho-1}\left|\delta_{\bar{z}}(\xi)-\vartheta_{z}(\xi)\right| d \xi+\frac{1}{\Gamma(\rho)} \int_{t_{i}}^{t}(t-\xi)^{\rho-1}|\phi(\xi)| d \xi \\
& +\frac{1}{\Gamma(\rho)} \sum_{i=1}^{m} \int_{t_{i-1}}^{t_{i}}\left(t_{i}-\xi\right)^{\rho-1}\left|\delta_{\bar{z}}(\xi)-\vartheta_{z}(\xi)\right| d \xi \\
& +\frac{1}{\Gamma(\rho)} \sum_{i=1}^{m} \int_{t_{i-1}}^{t_{i}}\left(t_{i}-\xi\right)^{\rho-1}|\phi(\xi)| d \xi \\
& +\frac{1}{\Gamma(\rho-1)} \sum_{i=1}^{m} \int_{t_{i-1}}^{t_{i}}\left(t_{i}-\xi\right)^{\rho-2}\left|\delta_{\bar{z}}(\xi)-\vartheta_{z}(\xi)\right| d \xi \\
& +\frac{1}{\Gamma(\rho-1)} \sum_{i=1}^{m} \int_{t_{i-1}}^{t_{i}}\left(t_{i}-\xi\right)^{\rho-2}|\phi(\xi)| d \xi
\end{aligned}
$$




$$
\begin{aligned}
& +\sum_{i=1}^{m}\left(t-t_{i}\right)\left|\tilde{\mathrm{I}}_{i}\left(\bar{z}\left(t_{i}\right)\right)-\tilde{\mathrm{I}}_{i}\left(z\left(t_{i}\right)\right)\right|+\sum_{i=1}^{m}\left(t-t_{i}\right)\left|\phi_{i}\right| \\
& +\sum_{i=1}^{m}\left|\mathrm{I}_{i}\left(\bar{z}\left(t_{i}\right)\right)-\mathrm{I}_{i}\left(z\left(t_{i}\right)\right)\right|+\sum_{i=1}^{m}\left|\phi_{i}\right| \\
& +\frac{1}{\Gamma(\rho)} \sum_{i=1}^{m+1} \int_{t_{i-1}}^{t_{i}}\left(t_{i}-\xi\right)^{\rho-1}\left|\delta_{\bar{z}}(\xi)-\vartheta_{z}(\xi)\right| d \xi \\
& +\frac{1}{\Gamma(\rho)} \sum_{i=1}^{m+1} \int_{t_{i-1}}^{t_{i}}\left(t_{i}-\xi\right)^{\rho-1}|\phi(\xi)| d \xi \\
& +\sum_{i=1}^{m}\left(1-t_{i}\right) \int_{t_{i-1}}^{t_{i}} \frac{\left(t_{i}-\xi\right)^{\rho-2}\left|\delta_{\bar{z}}(\xi)-\vartheta_{z}(\xi)\right|}{\Gamma(\rho-1)} d \xi \\
& +\int_{t_{i}}^{\varrho} \frac{(\varrho-\xi)^{\rho-2}\left|\delta_{\bar{z}}(\xi)-\vartheta_{z}(\xi)\right|}{\Gamma(\rho-1)} d \xi \\
& +\frac{1}{\Gamma(\rho-1)} \sum_{i=1}^{m}\left(1-t_{i}\right) \int_{t_{i-1}}^{t_{i}}\left(t_{i}-\xi\right)^{\rho-2}|\phi(\xi)| d \xi \\
& +\frac{1}{\Gamma(\rho-1)} \int_{t_{i}}^{\varrho}(\varrho-\xi)^{\rho-2}|\phi(\xi)| d \xi \\
& +\frac{1}{\Gamma(\rho-1)} \sum_{i=1}^{m} \int_{t_{i-1}}^{t_{i}}\left(t_{i}-\xi\right)^{\rho-2}\left|\delta_{\bar{z}}(\xi)-\vartheta_{z}(\xi)\right| d \xi \\
& +\frac{1}{\Gamma(\rho-1)} \sum_{i=1}^{m} \int_{t_{i-1}}^{t_{i}}\left(t_{i}-\xi\right)^{\rho-2}|\phi(\xi)| d \xi \\
& +\sum_{i=1}^{m}\left(1-t_{i}\right)\left|\tilde{\mathrm{I}}_{i}\left(\bar{z}\left(t_{i}\right)\right)-\tilde{\mathrm{I}}_{i}\left(z\left(t_{i}\right)\right)\right| \\
& +\sum_{i=1}^{m}\left(1-t_{i}\right)\left|\phi_{i}\right|+\sum_{i=1}^{m}\left|\tilde{\mathrm{I}}_{i}\left(\bar{z}\left(t_{i}\right)\right)-\tilde{\mathrm{I}}_{i}\left(z\left(t_{i}\right)\right)\right| \\
& +\sum_{i=1}^{n}\left|\phi_{i}\right|+\sum_{i=1}^{m}\left|\mathrm{I}_{i}\left(\bar{z}\left(t_{i}\right)\right)-\mathrm{I}_{i}\left(z\left(t_{i}\right)\right)\right|+\sum_{i=1}^{m}\left|\phi_{i}\right| .
\end{aligned}
$$

Thanks to $\left(\mathrm{H}_{1}\right)-\left(\mathrm{H}_{4}\right),(20)$ and part (i) of Remark 2, we have

$$
\begin{aligned}
|\bar{z}(t)-z(t)| \leq & \frac{L\|\bar{z}-z\|_{P C}}{\Gamma(\rho)(1-L)} \int_{t_{k}}^{t}(t-\xi)^{\rho-1} d \xi+\frac{2 L\|\bar{z}-z\|_{P C}}{\Gamma(\rho)(1-L)} \sum_{i=1}^{m+1} \int_{t_{i-1}}^{t_{i}}\left(t_{i}-\xi\right)^{\rho-1} d \xi \\
& +\frac{3 L\|\bar{z}-z\|_{P C}}{\Gamma(\rho-1)(1-L)} \sum_{i=1}^{m} \int_{t_{i-1}}^{t_{i}}\left(t_{i}-\xi\right)^{\rho-2} d \xi \\
& +\frac{L\|\bar{z}-z\|_{P C}}{\Gamma(\rho-1)(1-L)} \int_{t_{i}}^{\varrho}(\varrho-\xi)^{\rho-2} d \xi \\
& +2 m b\|\bar{z}-z\|_{P C}+3 m b^{*}\|\bar{z}-z\|_{P C}+\frac{\epsilon}{\Gamma(\rho)} \int_{t_{k}}^{t}(t-\xi)^{\rho-1} \theta(\xi) d \xi
\end{aligned}
$$




$$
\begin{aligned}
& +\frac{2 \epsilon}{\Gamma(\rho)} \sum_{i=1}^{m+1} \int_{t_{i-1}}^{t_{i}}\left(t_{i}-\xi\right)^{\rho-1} \theta(\xi) d \xi+\frac{3 \epsilon}{\Gamma(\rho-1)} \sum_{i=1}^{m} \int_{t_{i-1}}^{t_{i}}\left(t_{i}-\xi\right)^{\rho-2} \theta(\xi) d \xi \\
& +\frac{\epsilon}{\Gamma(\rho-1)} \int_{t_{i}}^{\varrho}(\varrho-\xi)^{\rho-2} \theta(\xi) d \xi+5 m \epsilon \varphi \\
\leq & \Upsilon\|\bar{z}-z\|_{P C}+\epsilon\left[\beta_{\theta}(5 m+4)+5 m\right](\varphi+\theta(t)) .
\end{aligned}
$$

This finally yields

$$
\begin{aligned}
\|\bar{z}-z\|_{P C} & \leq \frac{\left[\beta_{\theta}(5 m+4)+5 m\right]}{1-\Upsilon}(\varphi+\theta(t)) \epsilon, \\
\|\bar{z}-z\|_{P C} & \leq \mathbf{C}_{m, \rho} \epsilon(\varphi+\theta(t))
\end{aligned}
$$

where

$$
\mathbf{C}_{m, \rho}=\frac{\left[\beta_{\theta}(5 m+4)+5 m\right]}{1-\Upsilon} .
$$

Hence the solution to problem (1) is HUR stable and consequently GHUR stable.

\section{Example}

Consider the following implicit BVP of FODEs with impulsive conditions:

\section{Example 1}

$$
\left\{\begin{array}{l}
{ }_{0}^{C} \mathrm{D}_{t_{1}}^{\frac{3}{2}} z(t)=\frac{|z(t)|}{20(t+1)(1+|z(t)|)}+\frac{\sin \left|{ }_{0}^{C} \mathrm{D}_{t_{1}}^{\frac{3}{2}} z(t)\right|}{20 t^{2}}, \quad t \in \mathrm{J}, t \neq \frac{1}{3}, \\
\left.z(t)\right|_{t=0}=-\left.z^{\prime}(t)\right|_{t=0}, \quad z(1)=-z^{\prime}\left(\frac{1}{2}\right), \\
\Delta z\left(\frac{1}{3}\right)=\frac{\left|z\left(\frac{1}{3}\right)\right|}{60+\left|z\left(\frac{1}{3}\right)\right|}, \quad \Delta z^{\prime}\left(\frac{1}{3}\right)=\frac{\left|z\left(\frac{1}{3}\right)\right|}{45+\left|z\left(\frac{1}{3}\right)\right|} .
\end{array}\right.
$$

In this example, we see that $\rho=\frac{3}{2}, \varrho=\frac{1}{2}, m=1$. Set

$$
\left|\mathrm{F}\left(t, z(t), \delta_{z}(t)\right)\right|=\frac{|z(t)|}{20(t+1)(1+|z(t)|)}+\frac{\sin \left|{ }_{0}^{C} \mathrm{D}_{t_{1}}^{\frac{3}{2}} z(t)\right|}{20+t^{2}} .
$$

The continuity of $\mathrm{F}$ is obvious.

For $z, \bar{z} \in \mathrm{E}$ and $\delta_{z}, \delta_{\bar{z}} \in C(\mathrm{~J}, \mathrm{R}), t \in \mathrm{J}$,

$$
\left|\mathrm{F}\left(t, z(t), \delta_{z}(t)\right)-\mathrm{F}\left(t, \bar{z}(t), \delta_{\bar{z}}(t)\right)\right| \leq \frac{1}{20}\left(|z(t)-\bar{z}(t)|+\left|\delta_{z}(t)-\delta_{\bar{z}}(t)\right|\right) .
$$

This satisfies $\left(\mathrm{H}_{2}\right)$ with $K=L=\frac{1}{20}$. Further, for $t_{1}=\frac{1}{3}$, let

$$
\left.\Delta z(t)\right|_{t=t_{1}}=\frac{\left|z\left(t_{1}\right)\right|}{60+\left|z\left(t_{1}\right)\right|} \quad \text { and }\left.\quad \Delta(z(t))\right|_{t=t_{1}}=\frac{\left|z\left(t_{1}\right)\right|}{45+\left|z\left(t_{1}\right)\right|}, \quad \text { where } z \in \mathrm{E} \text {. }
$$

For any $z, \bar{z} \in \mathrm{E}$, we have

$$
\left|\mathrm{I}\left(z\left(t_{1}\right)\right)-\mathrm{I}\left(\bar{z}\left(t_{1}\right)\right)\right|=\left|\frac{\left|z\left(t_{1}\right)\right|}{60+\left|z\left(t_{1}\right)\right|}-\frac{\left|\bar{z}\left(t_{1}\right)\right|}{60+\bar{z}\left(t_{1}\right)}\right| \leq \frac{1}{60}\left|z\left(t_{1}\right)-\bar{z}\left(t_{1}\right)\right|
$$


and

$$
\left|\overline{\mathfrak{I}}\left(z\left(t_{1}\right)\right)-\overline{\mathfrak{I}}\left(\bar{z}\left(t_{1}\right)\right)\right|=\left|\frac{\left|z\left(t_{1}\right)\right|}{45+\left|z\left(t_{1}\right)\right|}-\frac{\left|\bar{z}\left(t_{1}\right)\right|}{45+\bar{z}\left(t_{1}\right)}\right| \leq \frac{1}{45}|z-\bar{z}| .
$$

These satisfy $\left(\mathrm{H}_{3}\right)$ and $\left(\mathrm{H}_{4}\right)$ with $b=\frac{1}{60}, b^{*}=\frac{1}{45}$.

Also

$$
\Upsilon=0.04355<1 .
$$

In view of Theorem 4, the uniqueness of solution to (22) follows. Thanks to Theorem 5 analogously one can see that the solution of problem (22) is HU stable and consequently GHU stable.

Further, assuming $\theta(t)=1$, we have

$$
{ }_{0} I_{t}^{\frac{3}{2}-1} \theta(t)=\frac{1}{\Gamma\left(\frac{3}{2}-1\right)} \int_{0}^{1}(1-s)^{\frac{3}{2}-2} s d s \leq \frac{1}{3 \sqrt{\pi}} .
$$

Thus $\left(\mathrm{H}_{8}\right)$ holds with $\beta_{\theta}=\frac{1}{3 \sqrt{\Pi}}$ and $\theta(t)=1$, therefore, in view of Theorem 6 , the solution of (22) is HUR stable corresponding to $(\theta, \varphi)$ and consequently GHUR stable with respect to $(\theta, \varphi)$.

\section{Conclusion}

By successful applications of nonlinear analysis and classical fixed point theory, we have developed adequate conditions under which the proposed class of implicit impulsive FODEs has at least one solution. Further, some useful results were also obtained that ensure different kinds of HUS which is important for the nonlinear problems from optimization and numerical point of view and plays a main role in numerical solutions where the exact solution is quite difficult.

\section{Acknowledgements}

We are really thankful to the reviewers for their useful suggestions and corrections.

Funding

This research work has been financially supported by Prof. Dumitru Baleanu of the Department of Mathematics, Cankaya University, Etimesgut/Ankara, Turkey.

\section{Abbreviations}

HUS, Hyers-Ulam stability; GHUS, Generalized Hyers-Ulam stability; HURS, Hyers-Ulam-Rassias stability; GHURS, Generalized Hyers-Ulam-Rassias stability.

Competing interests

The authors declare that they have no competing interests.

Authors' contributions

All authors equally contributed to this manuscript and approved the final version.

\section{Author details}

${ }^{1}$ Department of Mathematics, University of Malakand, Dir(L), Khyber Pakhtunkhwa, Pakistan. ${ }^{2}$ Department of Mathematics, Cankaya University, Ankara, Turkey.

\section{Publisher's Note}

Springer Nature remains neutral with regard to jurisdictional claims in published maps and institutional affiliations. 


\section{References}

1. Hilfer, R.: Applications of Fractional Calculus in Physics. World Scientific, Singapore (2000)

2. Kilbas, A.A., Srivastava, H., Trujillo, J.: Theory and Application of Fractional Differential Equations. North Holland Mathematics Studies, vol. 204. Elsevier, Amsterdam (2006)

3. Podlubny, I.: Fractional Differential Equations. Academic Press, New York (1993)

4. Khan, R.A., Shah, K.: Existence and uniqueness of solutions to fractional order multi-point boundary value problems. Commun. Appl. Anal. 19, 515-526 (2015)

5. Miller, B.M.: Method of discontinuous time change in problems of control for impulse and discrete-continuous systems. Autom. Remote Control 54(12), 1727-1750 (1993)

6. Sesekin, A.N., Zavalishchin, S.T.: Dynamic impulse systems: theory and applications. Math. Appl. 394, 4015-4025 (1997)

7. Shah, K., Khalil, H., Khan, R.A.: Investigation of positive solution to a coupled system of impulsive boundary value problems for nonlinear fractional order differential equations. Chaos Solitons Fractals 77, 240-246 (2015)

8. Lee, E.K., Lee, Y.H.: Multiple positive solutions of singular two point boundary value problems for second order impulsive differential equation. Appl. Math. Comput. 158, 745-759 (2004)

9. Lin, X., Jiang, D.: Multiple positive solutions of Dirichlet boundary value problems for second order impulsive differential equations. J. Math. Anal. Appl. 321, 501-514 (2006)

10. Lakshmikantham, V., Simeonov, P.S.: Theory of Impulsive Differential Equations. World scientific, Singapore (1989)

11. Shen, J., Wang, W.: Impulsive boundary value problems with nonlinear boundary conditions. Nonlinear Anal. 69, 4055-4062 (2008)

12. Wang, G., Zhang, L., Song, G.: Extremal solutions for the first order impulsive functional differential equations with upper and lower solutions in reversed order. J. Comput. Appl. Math. 235, 325-333 (2010)

13. Wang, J.R., Feckan, M., Zhou, Y.: A survey on impulsive fractional differential equations. Fract. Calc. Appl. Anal. 19(4), 806-831 (2016)

14. Tian, Y., Bai, Z: Existence results for the three-point impulsive boundary value problem involving fractional differential equations. Comput. Math. Appl. 59, 2601-2609 (2010)

15. Trigeassou, J.C., et al.: A Lyapunov approach to the stability of fractional differential equations. Signal Process. 91(3), 437-445 (2011)

16. Agarwal, R., Hristova, S., O'Regan, D.: A survey of Lyapunov functions, stability and impulsive Caputo fractional differential equations. Fract. Calc. Appl. Anal. 19(2), 290-318 (2016)

17. Stamova, l.: Mittag-Leffler stability of impulsive differential equations of fractional order. Q. Appl. Math. 73(3), 525-535 (2015)

18. Lijun, G., Wang, D., Wang, G.: Further results on exponential stability for impulsive switched nonlinear time-delay systems with delayed impulse effects. Appl. Math. Comput. 268, 186-200 (2015)

19. Ulam, S.M.: Problems in Modern Mathematics. Wiley, New York (1940)

20. Hyers, D.H.: On the stability of the linear functional equations. Proc. Natl. Acad. Sci. USA 27, 222-224 (1941)

21. Rassias, T.M.: On the stability of linear mappings in Banach spaces. Proc. Am. Math. Soc. 72, 297-300 (1978)

22. Jung, S.M.: Hyers-Ulam-Rassias Stability of Functional Equations in Nonlinear Analysis. Springer, New York (2011)

23. Ulam, S.M.: A Collection of Mathematical Problems. Interscience, New York (1968)

24. Fengqi, Y., Qiu, L., Shen, H.: On input to state stability of impulsive stochastic systems. J. Franklin Inst. 351(9), 4636-4651 (2014)

25. Rus, I.A.: Ulam stabilities of ordinary differential equations in a Banach space. Carpath. J. Math. 26, 103-107 (2010)

26. Shah, K., Ali, A., Bushnaq, S.: Hyers-Ulam stability analysis to implicit Cauchy problem of fractional differential equations with impulsive conditions. Math. Methods Appl. Sci. 41(17), 8329-8343 (2018)

27. Ali, A., Rabiei, F., Shah, K.: On Ulam's type stability for a class of impulsive fractional differential equations with nonlinear integral boundary conditions. J. Nonlinear Sci. Appl. 10(9), 4760-4775 (2017)

28. Cabada, A., Wang, G.: Positive solutions of nonlinear fractional differential equations with integral boundary value conditions. J. Math. Anal. Appl. 389(1), 403-411 (2013)

29. Granas, A., Dugundji, J.: Fixed Point Theory. Springer, New York (2003)

\section{Submit your manuscript to a SpringerOpen ${ }^{\circ}$ journal and benefit from:}

- Convenient online submission

- Rigorous peer review

- Open access: articles freely available online

- High visibility within the field

- Retaining the copyright to your article

Submit your next manuscript at $\boldsymbol{~ s p r i n g e r o p e n . c o m ~}$ 